\title{
Generalized elastodynamic model for nanophotonics
}

\author{
J. V. Alvarez, ${ }^{1}$ Bahram Djafari-Rouhani, ${ }^{2}$ and Dani Torrent ${ }^{3, *}$ \\ ${ }^{1}$ Departamento de Física de la Materia Condensada, Universidad Autónoma de Madrid, Condensed Matter \\ Physics Center (IFIMAC) and Instituto Nicolás Cabrera (INC), Madrid 28049, Spain \\ ${ }^{2}$ Institut d'Electronique, de Microléctronique et de Nanotechnologie, UMR CNRS 8520, Université de Lille, 59655 Villeneuve d'Ascq, France \\ ${ }^{3}$ GROC, UJI, Institut de Noves Tecnologies de la Imatge (INIT), Universitat Jaume I, 12071 Castelló, Spain
}

(Received 19 December 2019; revised 10 September 2020; accepted 15 September 2020; published 28 September 2020)

\begin{abstract}
A self-consistent theory for the classical description of the interaction of light and matter at the nanoscale is presented, which takes into account spatial dispersion. Up to now, the Maxwell equations in nanostructured materials with spatial dispersion have been solved by the introduction of the so-called additional boundary conditions which, however, lack generality and uniqueness. In this paper, we derive an approach where nonlocal effects are studied in a precise and uniquely defined way, thus allowing the treatment of all solid-solid interfaces (among metals, semiconductors or insulators), as well as solid-vacuum interfaces in the same framework. The theory is based on the derivation of a potential energy for an ensemble of electrons in a given potential, where the deformation of the ensemble is treated as in a solid, including both shear and compressional deformations, instead of a fluid described only by a bulk compressibility like in the hydrodynamical approach. The derived classical equation of motion for the ensemble describes the deformation vector and the corresponding polarization vector as an elastodynamic field, including viscous forces, from which a generalized nonlocal constitutive equation for the dielectric constant is derived. The required boundary conditions are identical to that of elastodynamics and they emerge in a natural way, without any physical hypothesis outside the current description, as is commonly required in other nonlocal approaches. Interestingly, this description does not require the discontinuity of any component of the electric, magnetic, or polarization fields and, consequently, no bounded currents or charges are present at the interface, which is a more suitable description from the microscopic point of view. It is shown that the method converges to the local boundary conditions in the low spatial dispersion limit for insulators and conductors, quantified by means of a parameter defined as the characteristic length. A brief discussion about the inclusion of the spill out of electrons across surfaces is discussed. Finally, the planar interface is studied and numerical examples of the behavior of the different fields at the interfaces are presented, showing the limiting situations in which the local limit is recovered, reinforcing the self-consistency of this description.
\end{abstract}

DOI: 10.1103/PhysRevB.102.115308

\section{INTRODUCTION}

The interaction of light and matter at the nanoscale has been a topic of intense research in recent years [1,2], due to the extraordinary advances in the manipulation capabilities of materials at the nanoscale [3-5]. The range of applications of this science is extremely broad and continues growing [6], therefore theoretical and numerical tools for their accurate description are of primary interest [7-9].

The nanoscale is a complex size limit for the study of the interaction of light and matter, since typical structures are big enough to consider the problem from the classical point of view, although some quantum effects can be observable. However, it is still possible to use a full classical description, as long as we find a proper constitutive equation relating the electric and magnetic fields with the induced currents and charges, which can take into account quantum corrections [10].

\footnotetext{
*dtorrent@uji.es
}

The simpler form of the constitutive equations used in electrodynamics are linear and local in both space and time, and they define the dielectric permittivity, magnetic permeability, and electrical conductivity [11]. Although nonlocality in time is commonly assumed, resulting in frequency-dependent constitutive parameters, spatial nonlocality is in general left behind to more refined models of matter, since they become important only at the nanoscale [12-15]. It has to be pointed out that in the domain of metamaterials, spatial dispersion has been a topic of intense research as well, since the distance between the meta-atoms is only one order of magnitude smaller than the operating wavelength of the field [16-22].

Despite the great success of the spatially local description at the macro or even microscales, the theory fails in the accurate description of nanomaterials, since spatial dispersion becomes more relevant and it has to be included in the constitutive parameters [23-30].

When the constitutive equations become spatially nonlocal, additional modes emerge in the solution of the wave equation, and the boundary conditions derived within the framework of Maxwell's equations are insufficient to match all the excited fields at an interface between two materials. 
This problem has derived in a countless number works proposal of the so-called additional boundary conditions, which are different depending on the different response models [30-34], which in turn means that spatial dispersion is not generally treated in the same way in insulators, semiconductors, or metals. Despite the fact that these additional boundary conditions are deduced with more or less reasonable physical assumptions, they are not derived within the framework of Maxwell's equations complemented with the constitutive equations, what means that the description is not "closed" and it can result in an inaccurate description of the electrodynamic problem, as can be seen for the fact that the problem still remains unsolved [35-37].

In this paper, we will show that, by means of an elastodynamic model of the induced current, we can find a self-consistent description of the light-matter interaction which accounts for spatial dispersion. We will show that when an ensemble of electrons is deformed, its potential energy is identical to that of an elastic body, so dynamically it moves as a continuous elastodynamic field [38]. In this context, we can develop a nonlocal theory for electrodynamics, where the required boundary conditions arise in a natural way. Moreover, we will define the local limit by means of a characteristic length parameter in which we recover the local description, therefore reinforcing the generality of this model.

The paper is organized as follows. After this Introduction, in Sec. II a discussion about the problem of nonlocality and its possible solutions is presented, then Sec. III presents the notions of quantum pressure and stress, and the elastic energy of the ensemble of electrons is derived. Section IV presents the elastodynamic formulation for nanophotonics, with a discussion about boundary conditions. Section V analyzes the solution of the wave equations in isotropic materials. Section VI discusses how this approach can include the spill out of electron across surfaces and Sec. VII compares the present approach with the hydrodyamic description. Section VIII analyzes the solid-solid and vacuum-solid interfaces. Finally, Sec. IX summarizes the paper.

\section{LOCAL AND NONLOCAL MATERIALS}

The evolution of the electromagnetic field inside a material where no sources are present is described by means of Maxwell's equations [11],

$$
\begin{gathered}
\nabla \times \boldsymbol{E}=-\partial_{t} \boldsymbol{B}, \\
\nabla \times \boldsymbol{B}=\mu_{0} \varepsilon_{0} \partial_{t} \boldsymbol{E}+\mu_{0} \boldsymbol{J}_{i}, \\
\nabla \cdot \boldsymbol{B}=0, \\
\nabla \cdot \boldsymbol{E}=\rho_{i} / \varepsilon_{0},
\end{gathered}
$$

where $\boldsymbol{J}_{i}$ and $\rho_{i}$ are the induced current and charge densities in the material, respectively. The continuity equation for the current density is implicit in Maxwell's equations and it is derived taking the divergence of Eq. (2) and using Eq. (4),

$$
\nabla \cdot \boldsymbol{J}_{i}+\partial_{t} \rho_{i}=0 .
$$

Maxwell's equations with the induced currents are not enough to solve the full electrodynamic problem, and we need a relationship between the induced current $\boldsymbol{J}_{i}$ and the electromagnetic field. This relationship is complex to obtain, since it implies a many-body problem solved in the framework of classical, semiclassical, or quantum theories, however, a phenomenological approach is commonly considered in which the classical permittivity, conductivity, and permeability are defined. Then, in classical electrodynamics, for nonmagnetic and nonchiral materials, the induced current is expressed as

$$
\boldsymbol{J}_{i}=\sigma_{E} \boldsymbol{E}+\varepsilon_{0} \chi_{E} \partial_{t} \boldsymbol{E},
$$

with $\sigma_{E}$ and $\chi_{E}$ being the electric conductivity and susceptibility, respectively. It is common to define the polarization vector $\boldsymbol{P}$ as

$$
\boldsymbol{J}_{i}=\partial_{t} \boldsymbol{P},
$$

so, assuming a harmonic time dependence of the fields of the form $\exp (-i \omega t)$, Eq. (6) is

$$
\boldsymbol{P}=\left(\varepsilon_{0} \chi_{E}+i \sigma_{E} / \omega\right) \boldsymbol{E},
$$

which defines the well-known complex dielectric constant $\varepsilon$ by means of the dielectric displacement $\boldsymbol{D}$, defined as

$$
\boldsymbol{D}=\varepsilon_{0} \boldsymbol{E}+\boldsymbol{P}=\varepsilon \boldsymbol{E},
$$

so

$$
\varepsilon=1+\chi_{E}+i \sigma_{E} /\left(\varepsilon_{0} \omega\right) .
$$

If either $\sigma_{E}$ or $\chi_{E}$ are discontinuous at an interface, so is the current $\boldsymbol{J}_{i}$ and, by means of Eq. (5), a surface charge appears. In classical electrodynamics, these discontinuities and surface fields are usual, and they do not imply any nonphysical description of the fields.

However, there are two points which make Eq. (6) unpleasant from a deeper physical insight. First, this local relationship in both time and space implies an instantaneous response of matter (locality in time) and a point-to-point response (locality in space), that is, the induced current in the material is instantaneous and it depends on the field at a given point only. Clearly, this is not a true physical situation, since the response of the charges will have some inertial response and will be influenced by the surrounding material. Another point, less discussed in the literature, is that current discontinuities and surface charges are not really possible at the microscopic level, since charges and currents are quantum entities described by wave functions which are in general continuous across the interfaces. Then, while it is true that the classical description of electrodynamics assumes that these discontinuities are just idealizations, we would like to find a description in which these do not occur so the theory will allow us to reduce more and more the scale of validity.

In this paper, we will show how, surprisingly, a nonlocal version of Eq. (6) is possible which additionally implies the continuity of all the fields involved in the interaction.

\section{A. Nonlocal constitutive equation}

When elementary models of the light-matter interaction are considered, the derived constitutive parameters $\chi_{E}$ and $\sigma_{E}$ are found to be frequency dependent, but this dependence has no 
consequences in the nature of the fields, since Eqs. (1)-(4) are usually solved assuming time-harmonic dependence.

However, the constitutive parameters can depend on the wave number as well, and this dependence is not equivalent to the frequency-dependence since, usually, we have to work on bounded materials. Then, let us assume, for instance, that the dielectric constant is both frequency and wave-number dependent. We will have (for the purposes of this section, we will consider only a scalarlike relation between fields, the full vectorial theory is developed later on)

$$
D(k, \omega)=\varepsilon(k, \omega) E(k, \omega),
$$

while we can continue working on the frequency-domain, if our material is bounded we need to work on the space domain and Fourier transform the above equation; thus we have

$$
D(x, \omega)=\int_{-\infty}^{\infty} \varepsilon\left(x-x^{\prime}, \omega\right) E\left(x^{\prime}, \omega\right) d x^{\prime},
$$

where we have used the convolution theorem. The above equation clarifies the nonlocal term for wave-numberdependent constitutive paramaters: the dielectric displacement at point $x$ depends linearly on the electric field at point $x^{\prime}$, distant from $x$ a length $d=x-x^{\prime}$. The dielectric function $\varepsilon(d, \omega)$, as a nonlocal response function, weights the contribution of electric fields at different $x^{\prime}$, and will decay as $d$ increases. In the local limit, this function is proportional to the Dirac delta function $\delta\left(x-x^{\prime}\right)$. It is obvious that if we introduce this relationship of the dielectric displacement with the electric field in Eqs. (1)-(4), even in the time-harmonic regime we obtain a more complicated equation. As the convolution theorem applies only to a spatially invariant medium, for a bounded material the dielectric constant will not be in general a function of $d=x-x^{\prime}$ and we get the most general linear relation between two scalar fields,

$$
D(x, \omega)=\int_{-\infty}^{\infty} \varepsilon\left(x, x^{\prime}, \omega\right) E\left(x^{\prime}, \omega\right) d x^{\prime},
$$

which complicates the problem even more. In next subsection, we will discuss how this problem could be solved in Fourier space by means of the so-called additional boundary conditions.

\section{B. The need for additional boundary conditions}

The usual method to solve the electrodynamic equations in bounded materials is by means of the application of boundary conditions, roughly speaking: knowing the solution of the fields in two materials, we apply boundary conditions at the interface between them and we find the solution of the problem. Maxwell's Eqs. (1)-(4) provide us of the required number of boundary conditions, once the constitutive equation for the induced current has been found, but only if this constitutive equation is local in space, otherwise we require additional boundary conditions, and these additional boundary conditions has been (and continue to be) a topic of a great discussion in the literature. The origin of this need is found in the number of solutions that a wave-number-dependent dielectric constant provides, as will be explained below.

Let us assume the problem of reflection and transmission at a flat interface. If the dielectric constant is frequency depen- dent only, the dispersion relation in the material is typically of the form

$$
k^{2}=\mu_{0} \varepsilon(\omega) \omega^{2},
$$

which means that, for a given frequency, we have two waves propagating through opposite directions (at normal incidence and in this scalar version, the vectorial case is more complex, as will be explained later). For a classical reflection and transmission problem, we will therefore have the incident wave, the reflected wave propagating backward, and the transmitted field at the other material. We will need to determine the amplitude of the reflected and transmitted waves and Maxwell equations indeed provide us two boundary conditions: the problem is perfectly defined.

However, if the dielectric constant is also wave-number dependent, for a given frequency our dispersion relation is

$$
k^{2}=\mu_{0} \varepsilon(\omega, k) \omega^{2},
$$

which, due to the dependence on $k$ of $\varepsilon$, can give more than two modes propagating in opposite directions. Then, a given incident field will excite, in the simpler of the situations, two reflected and two transmitted modes (assuming both materials nonlocal).

Maxwell equations provide only two boundary conditions but we have four modes to determine, so the problem is not well defined. We need the so-called additional boundary conditions to completely solve the problem.

The amount of works about these additional boundary conditions is huge and actually experts have developed the acronym $\mathrm{ABC}$ to refer to specific examples or generally, to the very issue presented above. There is no consensus about which ones are the correct ones. It has been assumed then that the correct boundary conditions depend on the microstructure of the materials and the interfaces, so there is not a unique solution for the problem of additional boundary conditions at the macroscopic level. However, we believe there is a well-defined macroscopic solution to this problem based on the correct definition of the constitutive parameters, whose specific values of course depend on the microstructure of the material but whose macroscopic behavior can be universally defined, as we do in the electrodynamics of local materials, where obviously we don't apply the same boundary conditions for metals and for lossless dielectrics, due to the fact that their constitutive parameters have different values. The next subsection will present the approach we will follow in this paper to overcome the problem of the additional boundary conditions.

\section{Solution from additional field equations}

The additional boundary conditions have been discussed in uncountable ways and these are suggested by more or less acceptable physical arguments or microscopical models. Our objective is to find a set of macroscopic equations from which we obtain the required boundary conditions, with these equations being functions of a set of (local) constitutive parameters whose numerical values define the different type of materials. It is obvious that the additional boundary conditions cannot be obtained from Maxwell equations, and physical arguments can be enough for simple situations (vacuum-solid interface at 
normal incidence) but more general situations require a more rigorous solution.

The motivation to search for the field equation of the induced current comes from Eq. (13), which is equivalent to a relationship between the polarization $\boldsymbol{P}$ and the electric field $\boldsymbol{E}$ of the form

$$
\boldsymbol{P}(\boldsymbol{r}, \omega)=\varepsilon_{0} \iiint \chi_{E}\left(\boldsymbol{r}, \boldsymbol{r}^{\prime}, \omega\right) \boldsymbol{E}\left(\boldsymbol{r}^{\prime}, \omega\right) d V .
$$

The above equation indeed suggests that the function $\chi_{E}\left(\boldsymbol{r}, \boldsymbol{r}^{\prime}, \omega\right)$ is the Green's function of the differential equation of the form

$$
\mathcal{L P}=\varepsilon_{0} \boldsymbol{E},
$$

and

$$
\mathcal{L} \chi_{E}\left(\boldsymbol{r}, \boldsymbol{r}^{\prime}, \omega\right)=\delta\left(\boldsymbol{r}-\boldsymbol{r}^{\prime}\right),
$$

with $\mathcal{L}$ being a partial differential operator. If we are able to find the operator $\mathcal{L}$, we will be able to find the correct boundary conditions in a similar way as we find them from Maxwell equations.

It has to be pointed out that the scope of this paper is not to model nor discuss the consequences of the frequency dependence of the complex dielectric constant, our objective is to discuss the consequences of the wave-number dependence, which will enter into the model through spatial derivatives in $\mathcal{L}$, and how this affects boundary conditions. Then, it has to be assumed that all the constants appearing in the model equations could present a more or less complex frequency dependence, even more if our material is artificially nanostructured, but we will not take care of this dependence.

\section{QUANTUM STRESS-STRAIN RELATION}

The most elementary microscopic model of the dielectric constant assumes that the electron is bounded to the atom by means of a springlike restoring force [11], so when the electric field interacts with it the equation of motion is

$$
m_{e} \ddot{\boldsymbol{r}}+\gamma \dot{\boldsymbol{r}}+\kappa \boldsymbol{r}=-e \boldsymbol{E},
$$

from which we can solve for the contribution to polarizability of one single electron as $\boldsymbol{p}=-e \boldsymbol{r}$. If the material is a conductor, the restoring force $-\kappa \boldsymbol{r}$ is set to zero and we recover Drude's model for the free electron. While these models are quite elementary, they allow us to explain some aspects of light-matter interaction, and in both models we recover a local and complex dielectric function.

\section{A. Quantum pressure and quantum stress}

Sommerfeld's model applies quantum statistics to the physics of the free electron in the solid, which is described as an ensemble of noninteracting spin- $1 / 2$ particles. It is found that an ensemble of $N$ particles in a box of volume $\Omega$ has a total energy $E$ given by

$$
E=\frac{3}{5} N E_{F},
$$

where $E_{F}$ is Fermi's energy and is the maximum energy level occupied by the electrons. This energy is proportional to $n^{2 / 3}$, with $n=N / \Omega$, so that we can obtain the so-called quantum pressure $\mathcal{P}$ of the gas from the thermodynamical definition of pressure,

$$
\mathcal{P}=-\left(\frac{\partial E}{\partial \Omega}\right)_{N}=\frac{2}{3} \frac{E}{\Omega},
$$

and the compressibility or bulk modulus:

$$
B=-\Omega \frac{\partial P}{\partial \Omega}=\frac{5}{3} \mathcal{P} .
$$

The electron gas is then described as a fluid material and the linearized equation of motion is that of the acoustic field subject to a body pressure due to the external electric field. This is the so-called hydrodynamic model for plasmonics [10] and, although widely used, we will show here that a more accurate description is needed, since the electron gas is found to have not only a bulk but also a shear modulus, so it is better described as a solid material.

Other models also include in this equation Ohmnic losses or diffusion [39], what essentially changes the frequency dependence of the constants involved in the model. Including other sources of dissipation at the quantum level, inter- or intraband transitions, for example [40], might also change this frequency response, but for the purpose of this paper it will be enough to assume that all the parameters appearing in the models are complex and frequency dependent, since our objective is not to model the interaction, but to understand the role and nature of the additional spatial derivatives appearing in the equation of motion for the induced current.

The hydrodynamic model assumes that the gas of electrons is a fluid and that only volumetric changes are possible. Moreover, it assumes as well that the internal restoring force is due to the gradient of a scalar pressure field. We will assume here a more general deformation of the ensemble of electrons to demonstrate that they actually behave as a solid.

Let us assume we have an ensemble of electrons in equilibrium in a periodic potential $V(\boldsymbol{r})$. Let us assume now that some external perturbation (like an electric field) modifies this wave function so a deformation $\boldsymbol{u}(\boldsymbol{r})$ is applied in such a way that the coordinates are transformed as

$$
\boldsymbol{r}^{\prime}=\boldsymbol{r}+\boldsymbol{u}(\boldsymbol{r}) .
$$

If a strain is defined in the usual way,

$$
\epsilon_{i j}=\frac{1}{2}\left(\frac{\partial u_{i}}{\partial x_{j}}+\frac{\partial u_{j}}{\partial x_{i}}\right),
$$

the stress of the deformed system is found as

$$
\sigma_{i j}=\frac{\partial \mathcal{E}}{\partial \epsilon_{i j}},
$$

with $\mathcal{E}$ being the elastic energy density. It is important to remark that, if rotations are excluded from the dynamics, the strain tensor is symmetric and the following property holds:

$$
\frac{\partial \epsilon_{k \ell}}{\partial \epsilon_{i j}}=\delta_{i k} \delta_{j \ell}+\delta_{i \ell} \delta_{j k}-\delta_{i j} \delta_{k \ell} .
$$

Let us assume now that the Hamiltonian of the system is given by

$$
\mathcal{H}=\sum_{\alpha}\left[\frac{\boldsymbol{p}_{\alpha}^{2}}{2 m_{\alpha}}+V\left(\boldsymbol{r}_{\alpha}\right)+\frac{1}{2} \sum_{\beta \neq \alpha} U\left(r_{\alpha \beta}\right)\right],
$$


where $\boldsymbol{p}_{\alpha}=-i \hbar \nabla_{\alpha}, \boldsymbol{r}_{\alpha}$ and $m_{\alpha}$ are the momentum, position and mass of the $\alpha$ particle, respectively. The term $V\left(\boldsymbol{r}_{\alpha}\right)$ stands for the periodic potential of the lattice. The term $U\left(r_{\alpha \beta}\right)$, with $r_{\alpha \beta}=\left|\mathbf{r}_{\alpha}-\mathbf{r}_{\beta}\right|$, is a two-body interaction between electrons.

Once the transformation is applied, each of these terms will contribute in a different way to the energy of the system. If a small deformation is assumed, the energy of the deformed system will be a function of the deformation $\boldsymbol{u}$ and the strain $\boldsymbol{\nabla} \boldsymbol{u}$. The difference between the energy of the deformed and undeformed systems is called the elastic energy, and it will allow us to define the elastic constants of the ensemble and to deduce the equation of motion.

\section{B. Kinetic term and the shear modulus of the free electron gas}

The kinetic energy of the deformed system, using the transformation $\partial_{i}^{\prime}=\partial_{i}-\partial_{j} u_{i} \partial_{j}$, is given by

$$
E_{K}=\frac{1}{2 m_{e}}\left\langle p^{2}-2 \frac{\partial u_{i}}{\partial x_{j}} p_{i} p_{j}+\frac{\partial u_{i}}{\partial x_{j}} \frac{\partial u_{i}}{\partial x_{k}} p_{j} p_{k}\right\rangle .
$$

The first term of the right-hand side of the above equation is the kinetic energy of the undeformed system,

$$
E_{K}^{0}=\left\langle\frac{\boldsymbol{p}^{2}}{2 m_{e}}\right\rangle,
$$

and the second term will be

$$
-\frac{1}{m_{e}}\left\langle\frac{\partial u_{i}}{\partial x_{j}} p_{i} p_{j}\right\rangle=-\left\langle\frac{p_{i}^{2}}{m_{e}}\right\rangle \frac{\partial u_{i}}{\partial x_{i}}=-\frac{2}{3} E_{K}^{0} \nabla \cdot \boldsymbol{u},
$$

where we have used the equipartition energy theorem:

$$
\left\langle p_{i}^{2} / 2 m\right\rangle=1 / 3\left\langle\boldsymbol{p}^{2} / 2 m\right\rangle .
$$

Finally, the last term is

$$
\left\langle\frac{1}{2 m_{e}} \frac{\partial u_{i}}{\partial x_{j}} \frac{\partial u_{i}}{\partial x_{k}} p_{j} p_{k}\right\rangle=\frac{1}{3} E_{K}^{0} \sum_{i, j}\left(\frac{\partial u_{i}}{\partial x_{j}}\right)^{2},
$$

so the contribution to the elastic energy of the kinetic part is given by

$$
E=-\frac{2}{3} E_{K}^{0} \nabla \cdot \boldsymbol{u}+\frac{1}{3} E_{K}^{0} \sum_{i, j}\left(\frac{\partial u_{i}}{\partial x_{j}}\right)^{2} .
$$

If rotations are ignored, the energy density can be expressed in terms of the strain as

$$
\mathcal{E}=\frac{E_{K}^{0}}{\Omega}\left(-\frac{2}{3} \epsilon_{i i}+\frac{1}{3} \epsilon_{i j} \epsilon_{i j}\right) .
$$

The volume $\Omega$ is also a function of the strain and is

$$
\Omega=\Omega_{0}\left(1+\epsilon_{j j}\right),
$$

with $\Omega_{0}$ being the volume of the undeformed system. The energy density is therefore given by

$$
\mathcal{E} \approx \frac{E_{K}^{0}}{\Omega_{0}}\left(-\frac{2}{3} \epsilon_{i i}+\frac{2}{3} \epsilon_{i i} \epsilon_{j j}+\frac{1}{3} \epsilon_{i j} \epsilon_{i j}\right),
$$

from which we can obtain the stress of the system using Eq. (25),

$$
\sigma_{i j}=\frac{\partial \mathcal{E}}{\partial \epsilon_{i j}}=\sigma_{i j}^{0}+C_{i j k \ell} \epsilon_{k \ell}
$$

where

$$
\sigma_{i j}^{0}=-\frac{2}{3} \frac{E_{K}^{0}}{\Omega_{0}} \delta_{i j}
$$

and

$$
C_{i j k \ell}=\frac{2}{3} \frac{E_{K}^{0}}{\Omega_{0}} \delta_{i j} \delta_{k \ell}+\frac{2}{3} \frac{E_{K}^{0}}{\Omega_{0}}\left(\delta_{i k} \delta_{j \ell}+\delta_{i \ell} \delta_{j k}\right) .
$$

If we consider only the kinetic part of the energy, we obtain the free electron gas, and it is shown that the unperturbed system is at a pressure $\mathcal{P}$ defined as

$$
\sigma_{i j}^{0}=-\mathcal{P} \delta_{i j},
$$

which, according to Eq. (38), is identical to that of the free electron gas derived from Fermi-Dirac statistics. However, Eq. (39) shows that the electron gas is not a fluid, but a solid with Lamé coefficients,

$$
\lambda_{S}=\mu_{S}=\mathcal{P},
$$

which actually gives the same bulk modulus of the free electron gas,

$$
B=\lambda_{S}+\frac{2}{3} \mu_{S}=\frac{5}{3} \mathcal{P},
$$

although the nature of the gas is not that of a fluid but of a solid, due to the presence of the shear modulus $\mu_{S}$. This result, already obtained in Refs. [41,42] using kinetic arguments, suggests that the hydrodynamic model, which ignores this shear modulus, can be refined. The electron gas actually moves as an isotropic solid, and its equation of motion should be that of elastodynamics. In the following two subsections, the interaction terms will be added which will consist of a body force due to the single-particle potential and a generalized stiffness tensor due to the two-body interaction.

\section{One-particle term}

Let us consider the contribution of the periodic potential to the elastic energy. Expanding the potential energy up to second order in the deformation, we obtain

$$
V\left(\boldsymbol{r}^{\prime}\right)=V(\boldsymbol{r})+\boldsymbol{u} \cdot \nabla V(\boldsymbol{r})+\frac{1}{2} u_{i} u_{j} \frac{\partial^{2} V(\boldsymbol{r})}{\partial x_{i} \partial x_{j}} .
$$

The first term of the above expression corresponds to the unperturbed system, the second and the third ones contribute therefore to the elastic energy. If we take the expected value of the energy we obtain

$$
E=-\nabla \cdot \boldsymbol{u}\langle V\rangle+\frac{1}{2} u_{i} u_{j}\left\langle V_{i j}\right\rangle,
$$

where we have integrated by parts for the first term but not in the second one. The energy density is therefore

$$
\mathcal{E} \approx-\frac{\langle V\rangle}{\Omega_{0}} \epsilon_{\ell \ell}+\frac{\langle V\rangle}{\Omega_{0}} \epsilon_{\ell \ell} \epsilon_{j j}+\frac{1}{2} u_{i} u_{j} \frac{\left\langle V_{i j}\right\rangle}{\Omega_{0}},
$$

where we have considered as well the variation of the volume $\Omega$ with the strain. We see then that the effect of the single-particle potential is to add a quantity $\langle V\rangle / \Omega_{0}$ to the equilibrium pressure $\mathcal{P}$ and to the Lamé parameter $\lambda_{S}$, while the shear modulus is not affected. Additionally, a term proportional to the square of the deformation appears, which 
will be the responsible of a body force density $f=-\nabla_{u} \mathcal{E}$, being

$$
f_{i}=-\frac{\left\langle V_{i j}\right\rangle}{\Omega_{0}} u_{j}
$$

This term is clearly the responsibility of the local dielectric function, and we would recover the classical oscillator model if we neglect the contribution of the nonlocal strain contribution.

\section{Two-body interaction}

The two-body interaction is traditionally introduced in the theory of phonons to derive the acoustic equation of motion, however, in our case the two-body interaction takes place between electrons, since we are interested in the optical regime where the nucleus will remain at rest. If we assume that the deformation is small, we have that

$$
\left|\boldsymbol{r}_{\alpha}^{\prime}-\boldsymbol{r}_{\beta}^{\prime}\right| \approx\left|\boldsymbol{r}_{\alpha}-\boldsymbol{r}_{\beta}\right|+\epsilon_{i j} \frac{\left(r_{i \alpha}-r_{i \beta}\right)\left(r_{j \alpha}-r_{j \beta}\right)}{\left|\boldsymbol{r}_{\alpha}-\boldsymbol{r}_{\beta}\right|} .
$$

Then, using $r_{\alpha \beta}=\left|\boldsymbol{r}_{\alpha}-\boldsymbol{r}_{\beta}\right|$, the two body potential is

$$
U\left(r_{\alpha \beta}^{\prime}\right) \approx U\left(r_{\alpha \beta}\right)+\epsilon_{i j} \frac{\partial U}{\partial \epsilon_{i j}}+\frac{1}{2} \epsilon_{i j} \epsilon_{k \ell} \frac{\partial^{2} U}{\partial \epsilon_{i j} \partial \epsilon_{k \ell}}
$$

with

$$
\frac{\partial U}{\partial \epsilon_{i j}}=\frac{\partial U}{\partial r_{\alpha \beta}} \frac{\partial r_{\alpha \beta}}{\partial \epsilon_{i j}}=\frac{\partial U}{\partial r_{\alpha \beta}} \frac{\left(\boldsymbol{r}_{\alpha \beta} \otimes \boldsymbol{r}_{\alpha \beta}\right)_{i j}}{r_{\alpha \beta}}
$$

and

$$
\frac{\partial^{2} U}{\partial \epsilon_{k \ell} \partial \epsilon_{i j}}=\frac{\partial^{2} U}{\partial r_{\alpha \beta}^{2}} \frac{\partial r_{\alpha \beta}}{\partial \epsilon_{i j}} \frac{\partial r_{\alpha \beta}}{\partial \epsilon_{k \ell}} .
$$

The two-body interaction therefore contributes with a fourthrank tensor to the stiffness of the system. Since this contribution has to be averaged through the unit cell and electrons are assumed to be Bloch wave functions, we expect this tensor to have the symmetries of the lattice.

\section{ELASTODYNAMIC FORMULATION}

In the previous section, we have shown that the elastic energy density of a deformed ensemble of electrons is given by

$$
\mathcal{E}=\sigma_{k \ell}^{0} \epsilon_{k \ell}+\frac{1}{2} C_{i j k \ell} \epsilon_{i j} \epsilon_{k \ell}+\frac{1}{2} \kappa_{i j} u_{i} u_{j},
$$

where the intrinsic stress of the system is

$$
\sigma_{i j}^{0}=-\left(\frac{2}{3} \frac{E_{K}^{0}}{\Omega_{0}}+\frac{\langle V\rangle}{\Omega_{0}}\right) \delta_{i j}+\frac{1}{2} \sum_{\alpha \neq \beta}\left\langle\frac{\partial U\left(r_{\alpha \beta}\right)}{\partial \epsilon_{i j}}\right\rangle,
$$

and the stiffness and body force tensors are given by

$$
\begin{aligned}
C_{i j k \ell}= & \left(\frac{2}{3} \frac{E_{K}^{0}}{\Omega_{0}}+\frac{\langle V\rangle}{\Omega_{0}}\right) \delta_{i j} \delta_{k \ell}+\frac{2}{3} \frac{E_{K}^{0}}{\Omega_{0}}\left(\delta_{i k} \delta_{j \ell}+\delta_{i \ell} \delta_{j k}\right) \\
& +\sum_{\alpha \neq \beta}\left\langle\frac{\partial^{2} U\left(r_{\alpha \beta}\right)}{\partial \epsilon_{k \ell} \partial \epsilon_{i j}}\right\rangle
\end{aligned}
$$

and

$$
\kappa_{i j}=\left\langle\frac{\partial^{2} V(\boldsymbol{r})}{\partial x_{i} \partial x_{j}}\right\rangle
$$

respectively. We have assumed, however, a time-independent deformation, for which the above energy is uniquely the potential energy of the system. To derive the classical equation of motion for the deformation, we have to assume that a kinetic energy term due to the time-variation of $\boldsymbol{u}$ will appear, so the Lagrangian density of the ensemble can be expressed as

$$
\mathcal{L}=\frac{1}{2} \rho_{M}\left(\frac{\partial \boldsymbol{u}}{\partial t}\right)^{2}-\sigma_{k \ell}^{0} \epsilon_{k \ell}-\frac{1}{2} C_{i j k \ell} \epsilon_{i j} \epsilon_{k \ell}-\frac{1}{2} \kappa_{i j} u_{i} u_{j},
$$

where $\rho_{M}$ is the mass density of the electron solid. Assuming a constant intrinsic stress results in the following equation of motion [43]:

$$
\rho_{M} \frac{\partial^{2} u_{i}}{\partial t^{2}}=\frac{\partial}{\partial x_{j}}\left(C_{i j k \ell} \frac{\partial u_{k}}{\partial x_{\ell}}\right)-\kappa_{i j} u_{j}+F_{i}^{e} .
$$

Since the induced polarization is $-\rho_{e} \boldsymbol{u}$ and the external force is due to the electric field, then $F_{i}^{e}=\rho_{e} E_{i}$, where $\rho_{e}$ is the charge density due to electrons, the above equation can be expressed as the system of equations

$$
\begin{gathered}
\rho_{M} \frac{\partial^{2} \boldsymbol{P}}{\partial t^{2}}=\nabla \cdot \sigma-\kappa \boldsymbol{P}+\rho_{M} \varepsilon_{0} \omega_{P}^{2} \boldsymbol{E}, \\
\sigma=\boldsymbol{C}: \nabla \boldsymbol{P},
\end{gathered}
$$

where we have used the definition of the plasma frequency $\varepsilon_{0} \omega_{P}^{2}=\rho_{e}^{2} / \rho_{M}$.

Finally, dissipation can be added phenomenologically to this model. The problem of dissipation is complex and an accurate description is beyond the scope of the present paper. It will, however, be considered in a phenomenological way. The local mechanism for dissipation is usually introduced by means of Ohms law, in which a finite conductivity appears so an induced current proportional to the electric field is excited.

However, dissipation in elastodynamics occurs in a different way, since for both fluids and solids it is due to forces proportional to the time derivatives of the strain. In our model, due to the presence of energy terms proportional to both the square of the strain and the deformation, we will assume that the two types of dissipation could appear, i.e., due to time derivatives of the strain and the deformation, what means that a local dissipative force $\boldsymbol{F}_{\gamma}=-\gamma \partial_{t} \boldsymbol{P}$ will appear in Eq. (57) and a viscous stress $\sigma_{\eta}=\boldsymbol{\eta}: \partial_{t} \boldsymbol{\nabla} \boldsymbol{P}$ will be added in Eq. (58), obtaining similar terms to those derived in Refs. [41,44].

In summary, the equation of motion of the electromagnetic field inside matter when no sources are present can now be described by means of the following set of equations:

$$
\begin{gathered}
\nabla \times \boldsymbol{E}=-\frac{\partial \boldsymbol{B}}{\partial t}, \\
\nabla \times \boldsymbol{B}=\mu_{0} \varepsilon_{0} \frac{\partial \boldsymbol{E}}{\partial t}+\mu_{0} \frac{\partial \boldsymbol{P}}{\partial t}, \\
\nabla \cdot \boldsymbol{B}=0, \\
\nabla \cdot\left(\varepsilon_{0} \boldsymbol{E}+\boldsymbol{P}\right)=0, \\
\rho_{M} \frac{\partial^{2} \boldsymbol{P}}{\partial t^{2}}=\nabla \cdot \sigma-\kappa \boldsymbol{P}-\gamma \frac{\partial \boldsymbol{P}}{\partial t}+\rho_{M} \varepsilon_{0} \omega_{P}^{2} \boldsymbol{E}, \\
\sigma=\boldsymbol{C}: \boldsymbol{\nabla} \boldsymbol{P}+\boldsymbol{\eta}: \partial_{t} \nabla \boldsymbol{P},
\end{gathered}
$$


where we have used in Eq. (62) the continuity equation

$$
\nabla \cdot P+\rho_{i}=0 .
$$

We can combine Eqs. (59) and (60) in the usual way to have a second-order partial differential equation relating $\boldsymbol{P}$ and $\boldsymbol{E}$. Similarly, we can combine Eqs. (64) and (63) and we will have another second-order partial differential equation relating $\boldsymbol{P}$ and $\boldsymbol{E}$. Combining these two, we will have a fourth-order partial differential equation, which in turn means that the eigenvalue problem for the fields will be a $3 \times 3$ matrix equation with a fourth power in the wave number, so in principle we will have three polarizations times the four solutions for the wave numbers, i.e., a total of 12 modes, but the restrictions due to Eqs. (61) and (62) reduces by two the number of modes, then the most general solution of Eqs. (59)-(64) in a homogeneous material will consist of ten propagating modes, but with double degeneracy due to reciprocity. This means that, at an interface between two different materials, we will need to match five modes at each side, so we will need ten boundary conditions. Equations (59)-(64) will allow us to derive these ten boundary conditions in a natural way, as will be discussed in the following section.

However, in some practical situations, one of the materials can be a local material, as is the case of vacuum, local dielectrics, or metals. In these situations, we will have less modes than boundary conditions, so we will need to find the boundary conditions that are no longer satisfied. This is opposite of the traditional approach in nonlocal electrodynamics, in which a great effort has been made trying to find additional boundary conditions. Clearly, this approach is more efficient, since going from the most general situation to particular cases is always easier than beginning with a particular case and trying to find the most general one, as will be seen in next section.

\section{A. Boundary conditions}

The set of Eqs. (59)-(64) define the evolution of the electromagnetic and polarization fields. Boundary conditions arise in a natural way in this description, since at an interface (flat or infinitesimally flat) the parallel wave number is a conserved quantity, and we can make in these equations the substitution $\boldsymbol{\nabla} \rightarrow \boldsymbol{n} \partial_{n}+i \boldsymbol{k}_{t}$. Since no singularities are allowed in any of the fields (no surface currents or fields), we impose the continuity of all the fields in front of the normal derivative $\partial_{n}$. It is easy to deduce then that the following conditions have to be satisfied at the boundary:

$$
\begin{aligned}
{\left[\boldsymbol{E}_{t}\right] } & =0, \\
{\left[\boldsymbol{B}_{t}\right] } & =0, \\
{[\boldsymbol{n} \cdot \sigma] } & =0, \\
{[\boldsymbol{P}] } & =0,
\end{aligned}
$$

where $[u] \equiv u^{+}-u^{-}$. The same conclusion could be reached with the traditional pill-box and circulation arguments. The first two equations are the well-known continuity equations of electrodynamics; the only difference is that in magnetic materials the microscopic field $\boldsymbol{B}_{t}$ has to be replaced by $\boldsymbol{H}_{t}$. The last two equations are identical to elastodynamics, where the continuity of the normal components of the stress tensor and the displacement vector are required.

The electrodynamic boundary conditions provide four equations (there are two components of each transverse field), while the elastodynamic ones provide us with six (the normal component of a second rank tensor is a three-component vector and the polarization vector has three components), therefore the above equations perfectly define the general boundary value problem at the interface between two materials, where ten equations were needed to match the five modes excited at each material, as explained before.

It is interesting to point out that within the elastodynamic description, the required boundary conditions are the continuity of the transverse components of the electric and magnetic fields; however, from Eq. (3), the continuity of the normal component of the magnetic field also holds, as usual, but also the continuity of the normal component of the electric field, since in Eq. (4) the continuity of the normal component of the polarization field also implies the continuity of the normal component of the electric field. The absence of discontinuities in the fields suggests that the elastodynamic description is more suitable for the study of nanostructured materials, where the continuous nature of the wave functions of the different polarization carriers also has to be imposed.

\section{B. Vacuum and local materials}

The boundary conditions derived in the previous subsection emerge in a natural way at the interface between two nonlocal materials. However, in many practical situations, one of the materials might be vacuum or a local material, in the sense that the effects of the stiffness tensor might be neglected. It will be also interesting to determine the conditions for the consideration of nonlocal effects, that is, the conditions under which the material cannot be considered a local material and this more complex theory has to be applied. We expect this limit happens for low frequencies, but these conditions will be derived later on. In this subsection, we will just consider what happens at an interface between a nonlocal material (solid) and a local one, which can be either vacuum or a local dielectric with finite conductivity, since, in terms of the number of solutions, all these materials are identical.

When one of the interfaces is vacuum or a local material, the number of modes to match reduces from ten to seven, since now we have the five modes of the solid material plus the two polarizations allowed in local electrodynamics. Therefore, we will also need seven boundary conditions for this interface.

Let us consider the local material first. We can assume that this situation will happen when no free or nearly free electrons are allowed (insulator), so the stiffness component due to the kinetic term cancels, and also when the two-body interaction between electrons is negligible, due to the fact that the restoring force constant $\kappa$ is so high that electrons remain bounded around the nucleus. The material is then polarizable only locally. At the interface, the electrons traveling through the solid find a very tiny potential barrier, so the electronic wave function will be continuous across the interface and, consequently, the polarization vector. However, it is clear that the free electrons from the solid will be able to apply a force at the boundary, but not the electrons at the local side, since 
the stress tensor is zero in the local material. In the case of local metals, these can be seen as those materials where the local dissipation term $\gamma$ is high, so the mean free path of the electrons is small. The effect is that the behavior of the material is local, since electrons are similarly "trapped" in a finite region, so that we will have a similar situation as described before.

Thus, the stress tensor can be discontinuous at this interface, and it is this boundary condition which is released here. We then have the following seven boundary conditions at the local-solid interface:

$$
\begin{aligned}
{\left[\boldsymbol{E}_{t}\right] } & =0, \\
{\left[\boldsymbol{B}_{t}\right] } & =0, \\
{[\boldsymbol{P}] } & =0 .
\end{aligned}
$$

It is now tempting to define vacuum as a "material" in which $\kappa$ is zero and also the stiffness tensor $C$. However, this would be the wrong picture of the vacuum-solid interface. Vacuum is indeed a region nonaccessible for the electrons of the solid, in the sense that these are bounded on its volume, we can consider therefore that it is the same situation as before but with the potential barrier found by the electrons in the solid being infinite, then it is the case when $\kappa \rightarrow \infty$ so we have to impose the cancellation of the wave function at its boundary and, equivalently, the cancellation of the polarization vector. This is indeed the same boundary condition as Eq. (67c), since in vacuum the polarization vector is zero and its continuity implies the cancellation at the boundary.

This is equivalent to the mechanically rigid body, in which we impose that the displacement of the surface be zero, so $\boldsymbol{P}=0$, while we are able to apply a force on its surface, meaning that $\sigma$ will be different than zero there. Then, boundary conditions will be

$$
\begin{aligned}
{\left[\boldsymbol{E}_{t}\right] } & =0, \\
{\left[\boldsymbol{B}_{t}\right] } & =0, \\
\boldsymbol{P} & =0 .
\end{aligned}
$$

The above boundary conditions are the ones used by Pekar[31], using the argument that the excitonic wave functions should be zero at a vacuum interface. In Sec. VIII, we will numerically show the above boundary conditions as a limiting case of $\kappa \rightarrow \infty$, and we will show numerically the transition from a nonlocal material to a local one.

The properties of plasmonic materials, when described by means of the hydrodynamical model [32], implies only one additional mode, the longitudinal one, thus it is not possible to cancel the three polarization components, and only the normal one is imposed. However, the justification of the third additional boundary condition is typically done by suggesting that since no charge is leaving the surface, we need that the normal component of the current and, therefore, of the polarization vector, has to be zero there. However, this is a tricky argument, since in the local description of metals with finite conductivity the normal component of the electric current is different than zero, and it does not mean that charges are leaving the surface, it means that we have charges on the surface. This is what happens indeed during the nonstationary regime of the charge of a capacitor.
In this unified picture of spatial dispersion, these boundary conditions arise in a more natural and general way, and we can assume that the normal component of the polarization is continuous in general and it cancels at the solid-bacuum interface, because vacuum is a rigid body and then its surface cannot be displaced.

\section{Final remarks about generalized boundary conditions}

It is the current view within the community working on nonlocal effects that boundary conditions cannot be uniquely established, mostly because they depend on the microscopic properties of each material and interface. Although this is certainly true for local materials, we propose to deal with the "microscopic" dependence of boundary conditions by including a few macroscopic quantities which help to establish the type of boundary in each interface. This approach essentially includes the strain tensor $\sigma$ and deduces a local relationship with the induced strain $\boldsymbol{\nabla} \boldsymbol{P}$. With this simple hypothesis, several response models are found. For instance, the different boundary conditions discussed in Refs. [33] or [37] can be derived by just changing the values of the stiffness tensor $\boldsymbol{C}$, local resonance $\omega_{R}$, plasma resonance $\omega_{P}$, and inertia $\rho_{M}$ on one side and the other of the interface; however, here boundary conditions are derived within the framework of the field equations, which is a more rigorous procedure. The statement "boundary conditions depend on the microstructure of the material" acquires a precise meaning in this context, since, obviously, the different values and symmetries of these parameters depend on the microstructure of the material. Limiting situations, like perfect conductors, are perfectly defined as particular situations where the boundary conditions are different.

The present formulation renounces the design of the constitutive parameters; it can be left behind to other domains of physics, and within the framework of this theory we can just study the physical properties of these materials assuming at least the order of magnitude of these parameters, but also imagine new phenomena and devices and guess which values of these constants would be required. Obviously, this is not the most general theory possible but, as we have found in the literature, it is a big step in the domain of nanophotonics since it unifies in just one model several materials widely studied.

The elastodynamic model is "closed" in the sense that we do not need additional physical considerations to properly define boundary conditions, while it is true that boundary conditions when spatial dispersion is present requires the knowledge of the microstructure of matter, it is also true that phenomenological theories have been derived in all domains of physics, where the different limiting values of the constitutive parameters can define different materials and responses, and this description seems a good step forward toward the general understanding of spatial dispersion at the nanoscale.

\section{SOLUTION IN ISOTROPIC MEDIA}

Although our approach is general and can be applied to any anisotropic material, we will illustrate the application of this method with the isotropic case. 
Let us assume that the fields have time-harmonic dependence of the form $\exp (-i \omega t)$, in this case the constitutive Eq. (64) defines a complex stiffness tensor which in isotropic media has only two independent components, then we have

$$
C_{i j m \ell}-i \omega \eta_{i j m \ell}=\alpha \delta_{i j} \delta_{m \ell}+\beta\left(\delta_{i m} \delta_{j \ell}+\delta_{j m} \delta_{i \ell}\right),
$$

where $\alpha$ and $\beta$ are complex parameters of the form $\alpha=\alpha_{R}-$ $i \omega \alpha_{I}$. The stress tensor is in this case given by

$$
\sigma_{i j}=\alpha \boldsymbol{\nabla} \cdot \boldsymbol{P} \delta_{i j}+\beta\left(\partial_{i} P_{j}+\partial_{j} P_{i}\right),
$$

and the equation of motion for $\boldsymbol{P}$ becomes

$$
(\alpha+\beta) \nabla \nabla \cdot \boldsymbol{P}+\beta \nabla^{2} \boldsymbol{P}-\Gamma \boldsymbol{P}=-\rho_{M} \varepsilon_{0} \omega_{P}^{2} \boldsymbol{E},
$$

where we have defined the frequency-dependent $\Gamma$ constant as $\Gamma=\rho_{M}\left(\omega_{R}^{2}-\omega^{2}\right)-i \gamma \omega$, with $\kappa=\rho_{M} \omega_{R}^{2}$. The above equation is equivalent to

$$
\begin{gathered}
{\left[(\alpha+2 \beta) \nabla^{2}-\Gamma\right] \nabla \cdot \boldsymbol{P}=-\rho_{M} \varepsilon_{0} \omega_{P}^{2} \nabla \cdot \boldsymbol{E},} \\
{\left[\beta \nabla^{2}-\Gamma\right] \nabla \times \boldsymbol{P}=-\rho_{M} \varepsilon_{0} \omega_{P}^{2} \nabla \times \boldsymbol{E},}
\end{gathered}
$$

which in Fourier space gives

$$
\begin{gathered}
\boldsymbol{k} \cdot \boldsymbol{P}=\frac{\rho_{M} \varepsilon_{0} \omega_{P}^{2}}{\Gamma+(\alpha+2 \beta) k^{2}} \boldsymbol{k} \cdot \boldsymbol{E}, \\
\boldsymbol{k} \times \boldsymbol{P}=\frac{\rho_{M} \varepsilon_{0} \omega_{P}^{2}}{\Gamma+\beta k^{2}} \boldsymbol{k} \times \boldsymbol{E},
\end{gathered}
$$

which allow us to solve for the polarization as

$$
\boldsymbol{P}=\frac{\rho_{M} \varepsilon_{0} \omega_{P}^{2}}{\Gamma+\beta k^{2}}\left[\boldsymbol{E}-\frac{\alpha+\beta}{\Gamma+(\alpha+2 \beta) k^{2}} \boldsymbol{k} \cdot \boldsymbol{E} \boldsymbol{k}\right] .
$$

If we divide the electric field in a polarization parallel $(\|)$ and perpendicular $(\perp)$ to the wave vector,

$$
\boldsymbol{E}=\boldsymbol{E}_{\|}+\boldsymbol{E}_{\perp},
$$

it is clear that we get

$$
\boldsymbol{P}=\epsilon_{0} \chi_{\|} \boldsymbol{E}_{\|}+\epsilon_{0} \chi_{\perp} \boldsymbol{E}_{\perp}
$$

with

$$
\begin{gathered}
\chi_{\|}(\omega, k)=\frac{\rho_{M} \omega_{P}^{2}}{\Gamma+(\alpha+2 \beta) k^{2}}, \\
\chi_{\perp}(\omega, k)=\frac{\rho_{M} \omega_{P}^{2}}{\Gamma+\beta k^{2}} .
\end{gathered}
$$

The dispersion relations and polarizations supported by the material can be found Fourier transforming Eqs. (60) and (59) so we get

$$
-\boldsymbol{k} \times \boldsymbol{k} \times \boldsymbol{E}=k_{0}^{2} \boldsymbol{E}+k_{0}^{2} / \varepsilon_{0} \boldsymbol{P}
$$

or

$$
-\boldsymbol{k} \cdot \boldsymbol{E} \boldsymbol{k}+k^{2} \boldsymbol{E}=k_{0}^{2} \boldsymbol{E}+k_{0}^{2} / \varepsilon_{0} \boldsymbol{P},
$$

from which we can solve for the longitudinal wave number

$$
k_{L}^{2}=-\rho_{M} \frac{\omega_{R}^{2}+\omega_{P}^{2}-\omega^{2}-i \gamma \omega}{\alpha+2 \beta},
$$

and the transverse one,

$$
\beta k_{T}^{4}-\left(\beta k_{0}^{2}-\Gamma\right) k_{T}^{2}-k_{0}^{2}\left(\Gamma+\rho_{M} \omega_{P}^{2}\right)=0,
$$

whose solutions are

$$
k_{T}^{2}=\frac{1}{2 \beta}\left[\beta k_{0}^{2}-\Gamma \pm \sqrt{\left.\left(\beta k_{0}^{2}+\Gamma\right)^{2}+4 \rho_{M} \omega_{P}^{2} \beta k_{0}^{2}\right)}\right],
$$

which shows that for real $\beta$ and $\Gamma$, i.e., with no dissipation, the squared wave number $k_{T}^{2}$ is always real, but can be negative or positive, therefore we will have either a propagating wave or a nondissipative evanescent one.

In summary, we have a longitudinal wave with wave number $k_{L}$ and two transverse modes with wave numbers $k_{T}$ given by the two solutions of Eq. (85). Taking into account that each transverse mode is decomposed in two polarizations, we have a total of five modes propagating through the bulk material in each direction, as discussed before.

\section{A. The quasilocal limit}

It is interesting to see that, in the absence of dissipation, if $\omega_{R}^{2}+\omega_{P}^{2}-\omega^{2}<0$, the longitudinal mode is propagative and also the two transverse modes $k_{T}$, since the product of the two solutions of Eq. (85) is

$$
k_{T 1}^{2} k_{T 2}^{2}=-\rho_{M} \frac{\omega_{R}^{2}+\omega_{P}^{2}-\omega^{2}}{\beta} .
$$

However, if $\omega_{R}^{2}+\omega_{P}^{2}-\omega^{2}>0$, only one of the transverse modes is propagative, and the longitudinal mode is also evanescent. We therefore have only two propagative modes and three evanescent ones. This is the situation that will be analyzed in this paper, since in terms of propagative modes the number of solutions is the same as in local materials, however, the role of the evanescent modes is to keep the continuity of the fields at the interface. Also, this situation will allow us to recover the local limit, as will be seen later, therefore we call this limit the quasilocal limit.

Then, in the limit $k_{0}^{2} \beta \rightarrow 0$, that is, the low frequency and low spatial dispersion limit, we can expand the solution for $k_{T}^{2}$ as

$$
k_{T}^{2} \approx \frac{\beta k_{0}^{2}-\Gamma}{2 \beta} \pm \frac{\beta k_{0}^{2}+\Gamma}{2 \beta}\left(1+\frac{2 \rho_{M} \omega_{P}^{2} \beta k_{0}^{2}}{\left(\Gamma+k_{0}^{2} \beta\right)^{2}}\right),
$$

which gives two physically different solutions, the propagating one (taking the plus sign in the \pm term), and hereafter labeled $a 1$,

$$
k_{a 1}^{2} \approx\left(1+\chi_{E}\right) k_{0}^{2},
$$

and the evanescent one, labeled $a 2$,

$$
k_{a 2}^{2} \approx-\frac{\rho_{M} \omega_{R}^{2}}{\beta} \equiv-\frac{1}{\ell_{0}^{2}},
$$

where we have defined the static electric susceptibility $\chi_{E}$ as

$$
\chi_{E}=\frac{\omega_{P}^{2}}{\omega_{R}^{2}-\omega^{2}-i \rho_{M}^{-1} \gamma \omega} .
$$

and the characteristic length $\ell_{0}$ as

$$
\ell_{0}=\frac{\beta}{\rho_{M} \omega_{R}^{2}} .
$$

Therefore, in this limit, the propagative solution is identical to that in a dielectric material with dielectric constant $1+\chi_{E}$, 
and evanescent modes will quickly be excited up to a distance $\ell_{0}$ from the surface.

The case of conductors is slightly different. A Drude metal is found in the limit of $\omega_{R} \rightarrow 0$, with $\gamma=0$, in the quasilocal limit we would have, assuming that $\omega_{P}^{2}>\omega^{2}$,

$$
k_{a 1}^{2} \approx\left(1-\frac{\omega_{P}^{2}}{\omega^{2}}\right) k_{0}^{2}<0,
$$

while in this case

$$
k_{a 2}^{2} \approx \frac{\rho_{M} \omega^{2}}{\beta},
$$

so even below the plasma frequency this model predicts the existence of a propagating mode at the speed of the electronic shear wave $c_{S}^{2}=\beta / \rho_{M}$. This velocity can be estimated in the following way: Since $\beta$ if of the order of the elastic constants of a solid, while $\rho_{M}$ is about 2000 times smaller than its mass density (the ratio between the proton and electron mass), we can estimate the speed of this wave being about 40 times faster than the speed of sound in solids. This additional mode will have a very short wavelength, so it will also be very quickly attenuated.

Indeed, if we consider the dissipation factor $\gamma$, we recover from $\chi_{E}$ the conductivity predicted by Drude model

$$
\sigma=\frac{\sigma_{0}}{1-i \omega \tau},
$$

with $\sigma_{0}=\rho_{e}^{2} \tau / \rho_{M}$ and $\tau=\rho_{M} / \gamma$, as expected, while for the second mode we have

$$
k_{a 2}^{2} \approx \frac{\omega^{2}}{c_{S}^{2}}\left(1+i \frac{1}{\omega \tau}\right)
$$

which gives

$$
k_{a 2} \approx \frac{\omega}{c_{S}}+\frac{i}{2 c_{S} \tau},
$$

so the length $\ell_{0}$ is now approximately given by the distance covered by the electrons traveling at the speed $c_{S}$, i.e., the shear speed of the ensemble. It will clearly be a short distance, and the local limit will be recovered, in this case taking the limit of this distance to zero, with the boundary conditions derived before, as will be demonstrated later on.

\section{B. The characteristic length $\ell_{0}$}

The term nonlocal has been used throughout this paper, paying special attention to the consequences in terms of wave propagation and the number of modes to match. Although this analysis is the relevant one in terms of the solution of boundary value problems, the analysis of the constitutive equations in real space is also interesting, since it will help us to better understand the physical interpretation of the stiffness tensor $\boldsymbol{C}$. We will limit our discussion to the isotropic case and neglecting the longitudinal response, but a deeper analysis can be found in Ref. [45].

For simplicity, we will assume that $\alpha=0$, so Eqs. (74) and (75) define a frequency and a wave-number-dependent electrical susceptibility,

$$
\boldsymbol{P}=\frac{\rho_{M} \omega_{P}^{2} \varepsilon_{0}}{\Gamma+\beta k^{2}} \boldsymbol{E} \equiv \varepsilon_{0} \chi_{N L}(k, \omega) \boldsymbol{E},
$$

which in real space relates the polarization and the electric field as

$$
\boldsymbol{P}(\boldsymbol{r}, \omega)=\varepsilon_{0} \iiint \chi_{N L}\left(\boldsymbol{r}-\boldsymbol{r}^{\prime}, \omega\right) \boldsymbol{E}\left(\boldsymbol{r}^{\prime}, \omega\right) d V,
$$

where the nonlocal susceptibility is

$$
\chi_{N L}(\boldsymbol{r}, \omega)=\rho_{M} \omega_{P}^{2} \iiint \frac{e^{i \boldsymbol{k} \cdot \boldsymbol{r}}}{\Gamma+\beta k^{2}} d V_{k},
$$

or, using the radial symmetry of the Fourier transform,

$$
\chi_{N L}(r, \omega)=\sqrt{\frac{2}{\pi}} \frac{\rho_{M} \omega_{P}^{2}}{r} \int_{0}^{\infty} \frac{k \sin k r}{\Gamma+\beta k^{2}} d k .
$$

Therefore, the polarization at a given point $r$ is a linear combination of the electric field through all the space weighted by the nonlocal susceptibility $\chi_{E}(\boldsymbol{r}, \omega)$. For both $\Gamma$ and $\beta$ real and positive (the quasidielectric limit), we have [see Ref. [46], Sec. 273, Eq. (3)]

$$
\chi_{N L}(r, \omega)=\sqrt{\frac{\pi}{2}} \frac{\rho_{M} \omega_{P}^{2}}{\beta} \frac{e^{-r / \ell_{0}}}{r}=\chi_{E} \sqrt{\frac{\pi}{2}} \frac{e^{-r / \ell_{0}}}{\ell_{0}^{2} r},
$$

where we have defined the characteristic length $\ell_{0}$ as

$$
\ell_{0}^{2}=\beta / \Gamma .
$$

The meaning of parameter $\ell_{0}$ is therefore clear in this limit: A region of radius $\ell_{0}$ has to be taken into account to compute the polarization $\boldsymbol{P}$, since outside this region the susceptibility $\chi_{N L}$ is negligible.

Once defined the characteristic length $\ell_{0}$, the effects of spatial dispersion will be more or less important depending on the wavelength of the field. As will be seen later, when $\lambda \gg \ell_{0}$ the influence region is averaged and the behavior of the fields is similar to that of a local material, while when $\lambda \approx \ell_{0}$ the effects of spatial dispersion are more relevant and cannot be ignored.

The above discussion is valid only in the quasilocal limit, that is, when all the additional modes are evanescent and we still have only one propagative solution. When either $\Gamma$ and $\beta$ are complex, the residue theorem has to be applied to evaluate Eq. (100), and we find that the exponential decay rate depends on the absolute value of the real part of $\sqrt{\Gamma / \beta}$. Then, let us assume for instance that $\beta$ is real, and $\Gamma=\rho_{M}\left(\omega_{R}^{2}-\omega^{2}\right)-$ $i \omega \gamma$, we will have

$$
\sqrt{\Gamma / \beta} \approx \sqrt{\frac{\rho_{M}\left(\omega_{R}^{2}-\omega^{2}\right)}{\beta}}\left(1-\frac{i \omega \gamma}{2 \rho_{M}\left(\omega_{R}^{2}-\omega^{2}\right)}\right) .
$$

The above result shows that as long as $\omega_{R}^{2}-\omega^{2}>0$, the characteristic length is of the order of $\ell_{0}$, as before, however, when $\omega_{R}^{2}-\omega^{2}<0$ the imaginary and real parts are exchanged, and the characteristic length becomes proportional to $1 / \delta$, which can be very high, depending on the value of this parameter. The same effect applies as well when $\beta$ has both real and imaginary parts, but not when it is purely imaginary. A deeper analysis of this complex situation is beyond the scope of the present paper, which is only to understand how the effects of spatial dispersion affects boundary conditions, therefore in the numerical calculations we will 
consider only the quasidielectric limit, in which the characteristic length is small and we can compare results with the local limit.

\section{SPILL OUT OF ELECTRONS}

In Sec. IV B, the boundary conditions for a vacuum-solid interface were discussed and vacuum was defined as a region nonaccessible for electrons, since the potential barrier between the solid and vacuum is assumed to be infinite. It can happen, however, that for some materials (insulators or conductors) this potential barrier can be finite, which allows the spill out of electrons from the solid to vacuum, as has been recently observed experimentally and discussed theoretically [9]. The spill out of electrons to vacuum can also be considered within the present model by means of two approaches, which will be discussed in the following two subsections. First, we will consider the situation where a small number of electrons leave the solid and then populate vacuum with a not necessarily uniform electron density $n_{0}$. Later we will discuss the solution by means of the density-functional theory (DFT), showing, however, that a more advanced approach is required in this case whose detailed solution is beyond the scope of the present paper.

\section{A. Homogeneous electron density}

Let us assume that some electrons leave the solid and they jump into vacuum, so now vacuum is a medium with some electron density $n_{0}$, which can be modelled as a perfect conductor, so in Eqs. (83) and (85) we set $\gamma=0$ and $\omega_{R}=0$. Additionally, if we assume that the number of electrons populating vacuum is small, we can also assume that $\omega_{P}=\rho_{e}^{2} / \varepsilon_{0} \rho_{M} \approx 0$, therefore the longitudinal wave number will be

$$
k_{L}^{2}=\frac{\rho_{M}}{\alpha+2 \beta} \omega^{2},
$$

while the two transverse modes will be, from Eq. (92),

$$
k_{a 1}=k_{0}
$$

and from Eq. (93):

$$
k_{a 2}^{2}=\frac{\rho_{M}}{\beta} \omega^{2} .
$$

Therefore, for this low-populated vacuum, we see that the electromagnetic field has likewise five modes, which correspond to the two transverse modes with wave number $k_{0}$ and, consequently, zero polarization, plus the three polarizations resulting from the solution of the elastodynamic Eq. (71), which are the two transverse modes plus the longitudinal one. We can interpret this low-populated medium as one in which the electromagnetic field is not really disturbed, so it propagates as in vacuum, however, the electrons are disturbed and they oscillate as a solid. There is obviously an electromagnetic field created by this polarization but it travels at a very small velocity.

\section{B. Inhomogeneous electron density}

The approach proposed in the previous section can be useful and accurate in the case of small gaps between solid layers, and it obviously requires an estimation of the density of electrons $n_{0}$ who have left the solids and are now at equilibrium in the gap. However, the electron density for a finite potential barrier will not be in general a continuous function, since the electron could have some complex spatial dependence $n_{0}=n_{0}(\boldsymbol{r})$. If the equilibrium electron density $n_{0}(\boldsymbol{r})$ is previously known, we can model the interaction by solving the field equations with the corresponding position-dependent parameters. Then, we know that for the free electron gas the $\alpha$ and $\beta$ parameters correspond to the Lamé constants and are given by [see Eq. (39)]

$$
\alpha(\boldsymbol{r})=\beta(\boldsymbol{r})=\mathcal{P}(\boldsymbol{r}),
$$

where the quantum pressure $\mathcal{P}(\boldsymbol{r})$ can be computed using Eq. (21) once the inhomogeneous equilibrium energy $E\left(n_{0}(\boldsymbol{r})\right)$ is known.

Boundary conditions remains the same but since the density of electrons $n_{0}(\boldsymbol{r})$ is a continuous function, we do not need to solve a boundary value problem, which somehow solves the problem of additional boundary conditions although closedform expressions for the scattering or transmission of waves are unlikely.

\section{Density and current functional theory}

In recent works, the above approach has been refined by means of the DFT, in which the density of electrons $n_{0}(\boldsymbol{r})$ is set as a new field variable and solved simultaneously with the field equations [47-49]. However, the DFT has been mainly developed for a scalar external potential, and the inclusion of the vector potential $\boldsymbol{A}$ in the interaction, which implies the definition of a current density functional theory (CDFT) is not as well developed as the DFT. It is easy to see then that, considering only the DFT, the movement of the electrons are assumed to be a gas or a fluid, so only they hydrodynamical model is suitable for this description. We see as well how within the framework of the DFT the hydrodynamical model can also be refined: We need the current as a functional as well . In Ref. [50], Ciracì applies CDFT to complement the hydrodynamical model and he uses a viscoelastic tensor to complement the equation of motion, using the expression derived in Ref. [41] but without adding the nonviscous term derived in this paper. We believe that the approach by Ciracì plus the elasticity tensor derived in this paper could represent a more accurate description than that derived within the frame work of the hydrodynamic model.

We can understand how the elastodynamic description could refine the application of DFT to plasmonics in the following way . Let us assume that the ensemble of electrons moves as a fluid material. Let us assume that, in equilibrium, some of the electrons can spill out from the solids to vacuum, and finally we have an equilibrium energy density $E\left(n_{0}(\boldsymbol{r})\right)$ as well as an equilibrium electron density $n_{0}(\boldsymbol{r})$, both inhomogeneous. If we assume that the energy $E$ is a functional solely of the density $n_{0}(\boldsymbol{r})$, any deviation from the equilibrium of the system will result in a restoring force due to the gradient of a pressure field $\mathcal{P}(\boldsymbol{r})$, derived from Eq. (21), thus the equation of motion of the ensemble will be

$$
\rho_{M} \frac{\partial \boldsymbol{J}}{\partial t}=\rho_{e}^{2} \boldsymbol{E}-\rho_{e} \nabla \mathcal{P}(\boldsymbol{r})
$$


However, a more general functional of the energy might imply that the energy be a function not only of the equilibrium density $n_{0}(\boldsymbol{r})$ but also of the equilibrium current $\boldsymbol{J}_{0}(\boldsymbol{r})$ and, consequently, the response of the ensemble to any deformation will be a restoring force due to the divergence of a stress field $\sigma_{i j}$, obtained from Eq. (25), thus the equation of motion will now be

$$
\rho_{M} \frac{\partial \boldsymbol{J}}{\partial t}=\rho_{e}^{2} \boldsymbol{E}+\rho_{e} \boldsymbol{\nabla} \cdot \sigma(\boldsymbol{r}) .
$$

Therefore, the only difference between the "classical" hydrodynamic formulation and the use of the DFT in the calculations is the equation of state relating the pressure field and the density $n_{0}(\boldsymbol{r})$; similarly, if we employ a Hook-like law for the stress $\sigma_{i j}$ above, we recover the elastodynamic formulation, while if we use the CDFT to obtain the stress from Eq. (25), we solve the full quantum-mechanical problem without the need of additional boundary conditions, since the only part missing is Maxwell equations and then the problem is self-contained.

If we exclude those effects related to microscopic details of the surface, we consider that the assumption of bounded domains with well-defined homogeneous electron densities describes the underlying physics of nonlocal materials. More microscopical techniques are obviously required in some extreme situations.

\section{FURTHER COMPARISON WITH THE HYDRODYNAMIC MODEL}

The theory we have presented in this paper can be applied to any solid (or fluid) material, since the interaction between electrons has been considered as well and it is a matter of obtaining the right constitutive parameters (with their corresponding symmetries) to properly model the optical response at the nanoscale. Nonlocal effects for plasmonic materials, where electrons are assumed to move freely through the conduction bands, have been studied mainly within the framework of the hydrodynamic model. The present model refines the hydrodynamic model, since it includes the shear modulus of the electron gas and not only the bulk modulus. The theoretical consequences of this refinement are summarized in this section and some numerical examples are given in Sec. VIII, which will serve as well as a guide for future experimental demonstrations to determine wether this refinement is required or not.

\section{A. Shear modulus}

As stated in Sec. III, the hydrodynamic model assumes that only an additional longitudinal mode exists and the equation of motion for the induced current is that of acoustics, with a bulk modulus $B$ defined by means of Eq. (22), while the present approach shows that for the free electron gas we have both a bulk modulus $B$ and a shear modulus $\mu_{S}$, i.e., the equation of motion for the induced current in the free electron gas is that of elastodynamics, where the shear modulus is given by Eq. (41), which shows that the relationship between the shear and bulk modulus is

$$
\mu_{S}=\frac{3}{5} B
$$

and, consequently, they are of the same order of magnitude and we should not neglect shear waves in this description. It is remarkable that we have derived Eq. (41) from a perturbation of the electronic wave function while in Ref. [41] the authors derived the same expressions using Boltzman's transport equation, which reinforces the accuracy of this result.

\section{B. Longitudinal and transverse dielectric functions}

Another remarkable argument in favor of the existence of this shear modulus is that, if taken into account, we obtain both a nonlocal longitudinal and transverse susceptibilities, as defined by Eqs. (79) and (80), while if we ignore the shear modulus, the perpendicular susceptibility becomes local and we have a nonlocal response only in the longitudinal component. However, this contradicts the result obtained with quantum mechanics using perturbation theory for the free electron gas, since it is shown there that the response function contains both longitudinal and transverse components [51].

\section{Nonlocal response of $S$-polarized waves}

Finally, the most interesting difference arising after including or not the shear modulus in the free electron gas is the fact that, according to the hydrodynamic description, the interaction of electromagnetic waves at any interface where the electric field is parallel to the surface (i.e., normal incidence in a plane or $S$ polarization in a plane or cylinder) will be indistinguishable from a local response. A nonlocal response in these situations can only be modeled (as will be shown in next section) if the shear modulus is included in the equation of motion. The nonlocal response of a material is due to the movement of electrons after the electromagnetic field has excited them; there is no obvious physical reason for which this response had to be local or nonlocal depending on the polarization of the field if the material is isotropic, and nonlocality is not only a surface effect, it is a bulk property that should not depend on the orientation of the field at the interface under consideration.

In the following section, we will show numerical results which will help us to understand when this difference with the hydrodynamic description is relevant or not, and it might be a guide for future considerations in the application of each description.

\section{NUMERICAL EXAMPLES: PLANAR INTERFACE}

In the previous sections, we have seen that the presence of shear forces in the movement of electrons as a continuum are found when considering both full quantum mechanical equations and the kinetic theory, which additionally are consistent with our elastodynamic approach. In this section, we will focus then on the nonlocal effects that the hydrodynamical cannot predict, i.e., those nonlocal effects related with the shear forces excited in the movement of electrons.

We will consider the planar interface between two solids, labeled $a$ and $b$, to analyze the consistency of the boundary conditions derived previously, as well as the conditions in which we recover the local limit. We will assume that material $a$ is at the left-hand side of the interface and material $\mathrm{b}$ is at the right-hand side, as shown in Fig. 1.

Let us assume that we are in the quasidielectric limit, in which one transverse wave number is propagative and the other transverse wave number is evanescent, as well as the 


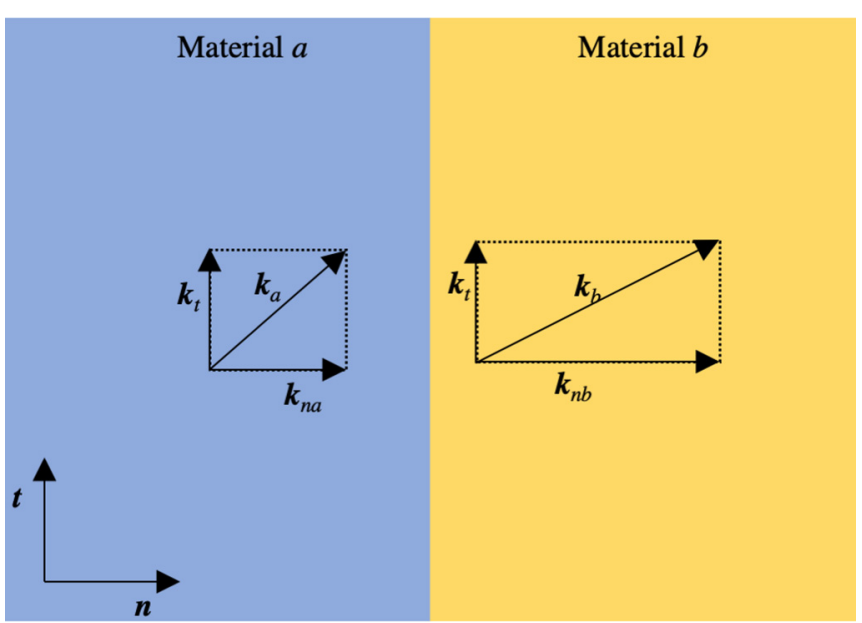

FIG. 1. Schematic diagram of the interface problem discussed in the text.

longitudinal one. It is convenient now to derive the relationship between the different fields involved in boundary conditions and the electric field. Thus, after performing the substitution $\boldsymbol{\nabla} \rightarrow \boldsymbol{i} \boldsymbol{k}_{t}+\partial_{n}$, from Eq. (59), we have that the transverse magnetic field is given by

$$
i \omega \boldsymbol{B}_{t}=\boldsymbol{n} \times \partial_{n} \boldsymbol{E}_{t}+i \boldsymbol{k}_{t} \times \boldsymbol{n} E_{n},
$$

while the polarization vector is given by

$$
\frac{k_{0}^{2}}{\varepsilon_{0}} \boldsymbol{P}=\left(k^{2}-k_{0}^{2}\right) \boldsymbol{E}+\left(\boldsymbol{n} \partial_{n}+i \boldsymbol{k}_{t}\right)\left(i \boldsymbol{k}_{t} \cdot \boldsymbol{E}_{t}+\partial_{n} E_{n}\right)
$$

The $i j$ element of the stress tensor $\sigma$ in an isotropic material is given in by Eq. (70), so the normal and transverse components, which are to be continuous, are

$$
\begin{gathered}
\sigma_{n n}=(\alpha+2 \beta) \partial_{n} P_{n}+i \alpha \boldsymbol{k}_{t} \cdot \boldsymbol{P}, \\
\sigma_{n t}=\beta \partial_{n} \boldsymbol{P}_{t}+i \beta \boldsymbol{k}_{t} P_{n} .
\end{gathered}
$$

In the next subsection, we will analyze the behavior of these fields at normal incidence, and later on we will derive the general reflection coefficients at the interface between vacuum and a solid material considering oblique incidence.

$$
\left(\begin{array}{ccc}
-1 & -1 & 1 \\
k_{a 1} & k_{a 2} & k_{b 1} \\
-\left(k_{a 1}^{2}-k_{0}^{2}\right) & -\left(k_{21}^{2}-k_{0}^{2}\right) & k_{b 1}^{2}-k_{0}^{2} \\
\beta_{a} k_{a 1}\left(k_{a 1}^{2}-k_{0}^{2}\right) & \beta_{a} k_{a 2}\left(k_{21}^{2}-k_{0}^{2}\right) & \beta_{b} k_{b 1}\left(k_{b 1}^{2}-k_{0}^{2}\right)
\end{array}\right.
$$

The above equations show that the interface problem is well defined once we have defined the two transverse wave numbers (at normal incidence there is no excitation of the longitudinal mode) and the $\beta$ coefficient. We will see now how the above equations, derived using Eq. (66), can degenerate in Eq. (67) as the limit of low characteristic length $\ell_{0}$.

\section{A. Normal incidence}

Let us assume that a propagating transverse wave is excited in material $a$ and propagates along the $x$ axis, so $\boldsymbol{n}=\hat{\boldsymbol{x}}$. Without loss of generality, we will assume that the interface is placed at $x=0$ and that the field is polarized along the $z$ direction. If the excited mode has wave vector $\boldsymbol{k}=k_{a 1} \hat{\boldsymbol{x}}$, after reflection two additional modes are excited in material $a$, corresponding to the two solutions of the dispersion Eq. (85). Since there are no normal components of the fields, there is no excitation of the longitudinal mode, whose discussion is left for the next subsection. Then we have, for $x<0$,

$$
E_{z}=e^{i k_{a 1} x}+R_{1} e^{-i k_{a 1} x}+R_{2} e^{-i k_{a 2} x} .
$$

The transverse component of the magnetic field is

$$
\left.\omega B_{y}=k_{a 1}\left(e^{i k_{a 1} x}-R_{1} e^{-i k_{a 1} x}\right)-k_{a 2} R_{2} e^{-i k_{a 2} x}\right),
$$

and the polarization vector is parallel to the electric field, therefore

$$
\frac{k_{0}^{2}}{\varepsilon_{0}} P_{z}=\left(k_{a 1}^{2}-k_{0}^{2}\right)\left(e^{i k_{a 1} x}+R_{1} e^{-i k_{a 1} x}\right)+\left(k_{a 2}^{2}-k_{0}^{2}\right) R_{2} e^{-i k_{a 2} x} .
$$

The normal component of the stress tensor $\sigma$ is zero, and the transverse one is

$$
\begin{aligned}
\frac{\varepsilon_{0}}{i k_{0}^{2}} \sigma_{z x}= & \beta_{a} k_{a 1}\left(k_{a 1}^{2}-k_{0}^{2}\right)\left(e^{i k_{a 1} x}-R_{1} e^{-i k_{a 1} x}\right) \\
& -\beta_{a} k_{a 2}\left(k_{a 2}^{2}-k_{0}^{2}\right) R_{2} e^{-i k_{a 2} x} .
\end{aligned}
$$

For $x>0$, we have two modes excited as well, thus

$$
\begin{gathered}
E_{z}=T_{1} e^{i k_{b 1} x}+T_{2} e^{i k_{b 2} x} \\
\omega B_{y}=k_{b 1} T_{1} e^{i k_{b 1} x}+k_{b 2} T_{2} e^{i k_{b 2} x} \\
\frac{k_{0}^{2}}{\varepsilon_{0}} P_{z}=\left(k_{b 1}^{2}-k_{0}^{2}\right) T_{1} e^{i k_{b 1} x}+\left(k_{b 2}^{2}-k_{0}^{2}\right) T_{2} e^{i k_{b 2} x} \\
\frac{\varepsilon_{0}}{i k_{0}^{2}} \sigma_{z x}=\beta_{b} k_{b 1}\left(k_{b 1}^{2}-k_{0}^{2}\right) T_{1} e^{i k_{b 1} x}-\beta_{b} k_{b 2}\left(k_{b 2}^{2}-k_{0}^{2}\right) T_{2} e^{i k_{b 2} x} .
\end{gathered}
$$

In summary, we will need to determine the value of four coefficients, and we have indeed four equations for this geometry, the continuity of $E_{z}, B_{y}, P_{z}$, and $\sigma_{z x}$, then the solution for the coefficients can be found after inversion of the system:

$$
\left.\begin{array}{c}
1 \\
k_{b 2} \\
k_{b 2}^{2}-k_{0}^{2} \\
\beta_{b} k_{b 2}\left(k_{b 2}^{2}-k_{0}^{2}\right)
\end{array}\right)\left(\begin{array}{c}
R_{1} \\
R_{2} \\
T_{1} \\
T_{2}
\end{array}\right)=\left(\begin{array}{c}
1 \\
k_{a 1} \\
\left(k_{a 1}^{2}-k_{0}^{2}\right) \\
\beta_{a} k_{a 1}\left(k_{a 1}^{2}-k_{0}^{2}\right)
\end{array}\right) .
$$

Figure 2 shows the polarization $P(x)$ (upper panel) and the stress tensor $\sigma_{x z}$ as a function of $x$ along an interface between two solid materials. Material $a$ is chosen $\operatorname{so} \beta_{a}=3, k_{a 1}^{2}=(1+$ $\left.\chi_{E a}\right) k_{0}^{2}$ and $k_{a 2}^{2}=-1 / \ell_{a}^{2}$, with $\chi_{E a}=2$ and $\ell_{a}=\lambda / 10$. Similarly, material $b$ is selected as $\beta_{b}=1, k_{b 1}^{2}=\left(1+\chi_{E b}\right) k_{0}^{2}$ and $k_{b 2}^{2}=-1 / \ell_{b}^{2}$, with $\chi_{E b}=3$ and $\ell_{b}=\lambda / 10, \lambda / 50$ and $\lambda / 100$. 

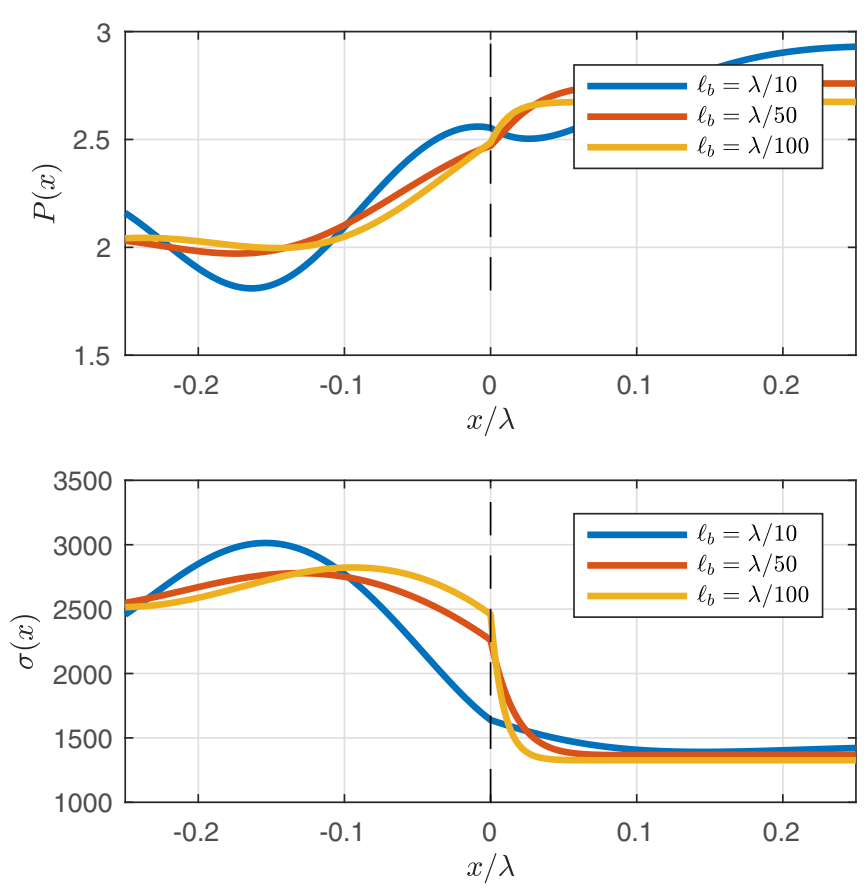

FIG. 2. Polarization vector (upper panel) and stress field (lower panel) as a function of $x$ near an interface solid-solid for several values of the characteristic length $\ell_{b}$ of material $b$. We see how in the transition $\ell_{b} \rightarrow 0$ material $b$ becomes a local material and the stress tensor presents a discontinuity, as predicted by the theory (see text for further details).

We see that both solids are nonlocal but we have analyzed the evolution of material $b$ toward a local material, showing therefore that in this transition the polarization vector remains continuous while the stress tensor presents a discontinuity, as required by boundary conditions Eq. (67).

Figure 3 shows the same situation as before but now we set $\chi_{E b}$ equal to 0 , so in the limit of $\ell_{b} \rightarrow 0$ material $a$ converges towards vacuum. We see that, as expected for vacuum, both the polarization and the stress fields cancels, however, the polarization remains continuous (zero) at the interface while the stress presents a discontinuity. Once more, this agrees with boundary conditions Eq. (68).

Figure 4 shows the same situation but now we set $\chi_{E b}=-100$, which corresponds to a metallic material. The meaning of the characteristic length $\ell_{b}$ is similar, as shown in Eq. (96), but now we add the contribution of the short wave to $k_{b 2}$, so we have $k_{b 2}=k_{S}+i / \ell_{b}$, with $k_{S}=10 k_{0}$ and $\ell_{b}$ having the same range as before.

Finally, Fig. 5 shows the behavior of the polarization vector at a vacuum-solid interface, when the solid has a dielectric (upper panel) or a conductor (lower panel) character, with parameters $\beta_{b}=2, \chi_{E b}= \pm 3$, with the $+(-)$ sign corresponding to the dielectric (conductor). For the conductor case, we also set $k_{S}=10 k_{0}$ and results are shown for $\ell_{b}=$ $\lambda / 10, \lambda / 25, \lambda / 50$, and $\lambda / 100$. Boundary conditions imply the cancellation of the polarization at the interface, but we see how, as the characteristic length becomes smaller, we recover the local behavior for both types of materials, and the polarization presents a step discontinuity for the dielectric while it is concentrated at the surface for the conductor. It has to
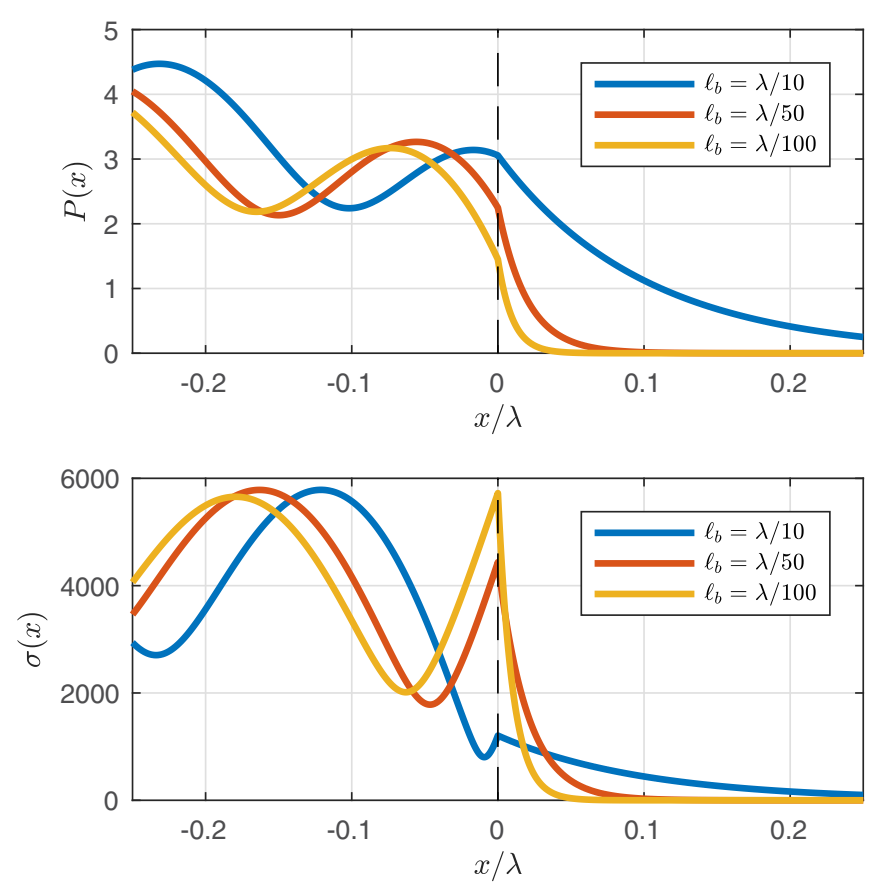

FIG. 3. Same system as described in Fig. 2 but now material $b$ converges toward vacuum.

be pointed out that, within the hydrodynamic description, the behavior of the conductor could not be described in this way, since at normal incidence it is identical to a local metal.

The presented approach is clearly consistent in the limiting situations, in which we recover the "traditional" boundary conditions as a progressive limiting situation, something that is not given in other approaches, which just define a set of
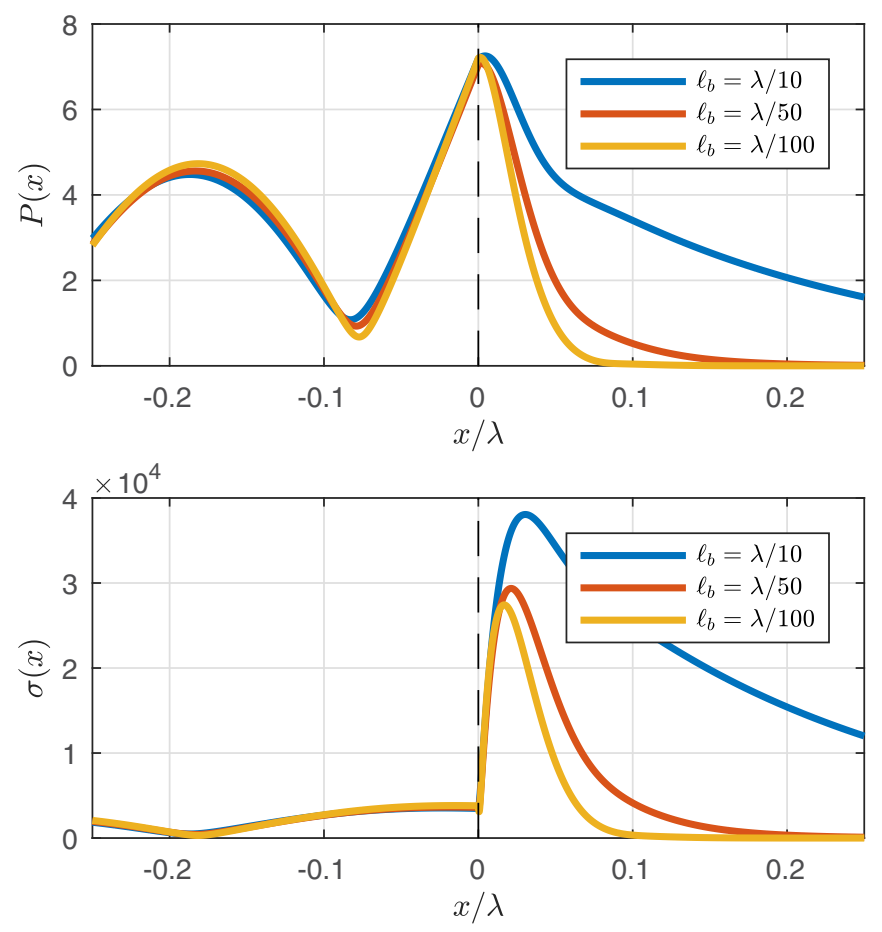

FIG. 4. Same situation as in Fig. 2 but now material $b$ is a metal with $\chi_{E b}=-100$ and $k_{b 2}=k_{S}+i / \ell_{b}$. 

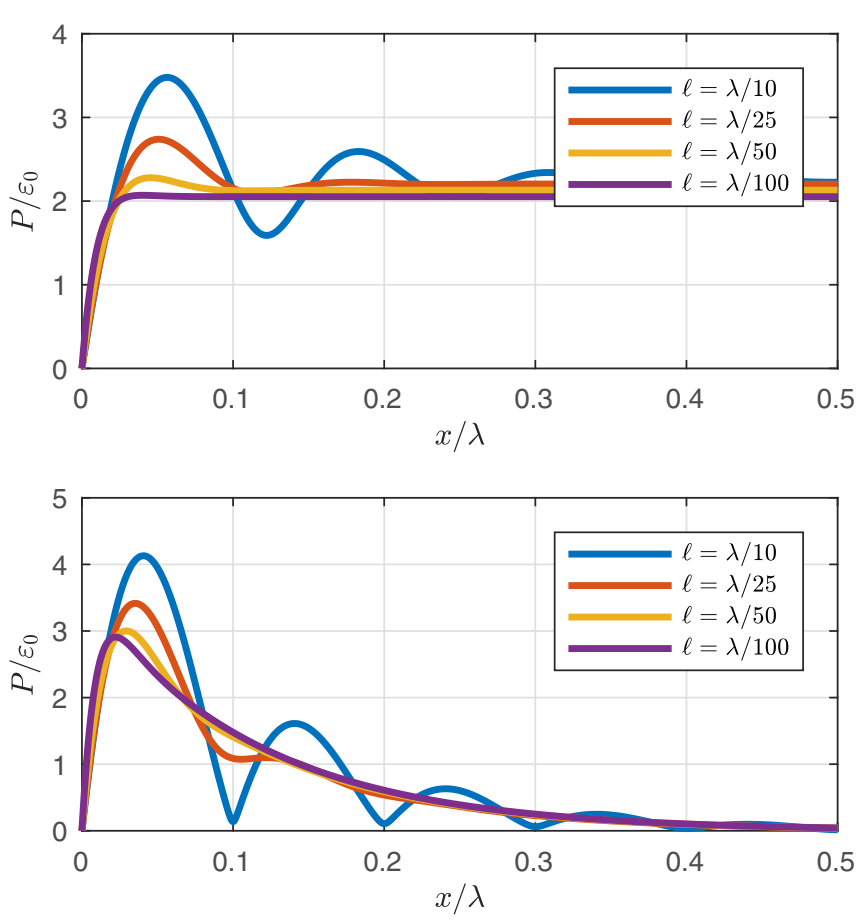

FIG. 5. Polarization vector in a vacuum-solid interface when the solid is a nonlocal dielectric (upper panel) or conductor (lower panel). We see how, although the polarization cancels at the surface, as we reduce the characteristic length the polarization tends toward a step discontinuity in the case of the nonlocal dielectric and to concentrate all the current at the surface for the conductor, as predicted by local electrodynamics but also in agreement with the present theory.

boundary conditions but do not specify when these are valid or not.

\section{B. Oblique incidence}

In the previous subsection, we considered the different interfaces at normal incidence, and this analysis allowed us to study the behavior of the fields when the material's discontinuities appear. In most of the real problems, the fields do not incide normally at a surface, as, for example, in cylindrical or spherical objects, therefore the analysis of the behavior of the fields when a given angle $\theta_{0}$ is formed with the normal to the surface will help us to understand the influence of spatial dispersion in more general problems. As will be seen below, the main difference is that the longitudinal mode is excited when a normal component of the electric field appears at the interface. This longitudinal mode will be required to satisfy the continuity of the fields and it will play a similar role as the evanescent mode studied before.

For an isotropic material, the possible solutions of the electromagnetic field can be decomposed in one longitudinal $(L)$ and two transverse $(T)$ modes, and each of the transverse modes has two components, which we define as the $S_{1}, S_{2}$ and $P_{1}, P_{2}$ polarizations.

Since the component of the wave vector parallel to the interface is a conserved quantity and, consequently, identical to all the polarizations, we define the wave vector as

$$
\boldsymbol{k}_{i \sigma}^{ \pm}= \pm q_{i \sigma} \boldsymbol{n}+\boldsymbol{k}_{t},
$$

where the index $i$ indicates in which material the wave propagates $\left(i=a, b\right.$ in our case) and $\sigma=L, S_{1}, S_{2}, P_{1}, P_{2}$. The vector $\boldsymbol{k}_{t}$ is the component of the wave vector parallel to the surface, therefore the component of the wave vector normal to the surface is given by

$$
q_{i \sigma}=\sqrt{k_{i \sigma}^{2}-\left|\boldsymbol{k}_{t}\right|^{2}}
$$

with $k_{i \sigma}=\left|\boldsymbol{k}_{i \sigma}^{ \pm}\right|$being $k_{L}$ for $\sigma=L$ and $k_{T}$ for the different transverse modes, i.e., the solutions of Eqs. (83) and (85).

The unit vectors parallel to these polarizations are given by

$$
\begin{gathered}
\boldsymbol{u}_{L}=\frac{\boldsymbol{k}_{L}}{\left|\boldsymbol{k}_{L}\right|}, \\
\boldsymbol{u}_{S}=\boldsymbol{n} \times \frac{\boldsymbol{k}_{T}}{\left|\boldsymbol{k}_{T}\right|}, \\
\boldsymbol{u}_{P}=\boldsymbol{u}_{S} \times \frac{\boldsymbol{k}_{T}}{\left|\boldsymbol{k}_{T}\right|},
\end{gathered}
$$

and the following relationships are found:

$$
\begin{gathered}
\boldsymbol{k}_{L} \times \boldsymbol{u}_{L}=0 . \\
\boldsymbol{k}_{T} \times \boldsymbol{u}_{S}=k_{T} \boldsymbol{u}_{P}, \\
\boldsymbol{k}_{T} \times \boldsymbol{u}_{P}=-k_{T} \boldsymbol{u}_{S} .
\end{gathered}
$$

It has to be pointed out that the $\boldsymbol{u}_{S}$ and $\boldsymbol{u}_{P}$ are orthogonal for the same root of Eq. (85) but not for different roots. Also, $\boldsymbol{u}_{L}$ is not in general orthogonal to these vectors.

We assume that material $a$ is vacuum and that the incident electric field is a plane wave with a general polarization state, thus

$$
\boldsymbol{E}_{0}=\sum_{\sigma=S, P} A_{\sigma} e^{i q_{a \sigma} n} e^{i \boldsymbol{k}_{t} \cdot \boldsymbol{r}} \boldsymbol{u}_{\sigma}^{+},
$$

which excites a reflected field given by

$$
\boldsymbol{E}_{R}=\sum_{\sigma=S, P} B_{\sigma} e^{-i q_{a \sigma} n} e^{i \boldsymbol{k}_{t} \cdot \boldsymbol{r}} \boldsymbol{u}_{\sigma}^{-} .
$$

Material $b$ is a nonlocal solid, therefore we will have two solutions for each of the $S$ and $P$ polarizations plus the longitudinal mode $L$, therefore the transmitted electric field will be

$$
\boldsymbol{E}_{T}=\sum_{\sigma=S_{j}, P_{j}, L} C_{\sigma} e^{i q_{b \sigma} n} e^{i \boldsymbol{k}_{t} \cdot \boldsymbol{r}} \boldsymbol{u}_{\sigma}^{+},
$$

where $\sigma=S_{1}, S_{2}, P_{1}, P_{2}$, and $L$. Related with each component of the electric field, we have the magnetic field

$$
\begin{gathered}
\omega \boldsymbol{B}_{0}=\sum_{\sigma=S, P} A_{\sigma} e^{i q_{a \sigma} n} e^{i \boldsymbol{k}_{t} \cdot \boldsymbol{r}} \boldsymbol{k}_{a \sigma}^{+} \times \boldsymbol{u}_{\sigma}^{+}, \\
\omega \boldsymbol{B}_{R}=\sum_{\sigma=S, P} B_{\sigma} e^{-i q_{a \sigma} n} e^{i \boldsymbol{k}_{t} \cdot \boldsymbol{r}} \boldsymbol{k}_{a \sigma}^{-} \times \boldsymbol{u}_{\sigma}^{-}, \\
\omega \boldsymbol{B}_{T}=\sum_{\sigma=S_{j}, P_{j}, L} C_{\sigma} e^{i q_{b \sigma} n} e^{i \boldsymbol{k}_{t} \cdot \boldsymbol{r}} \boldsymbol{k}_{b \sigma}^{+} \times \boldsymbol{u}_{\sigma}^{+},
\end{gathered}
$$


and the polarization vector

$$
\begin{aligned}
-\frac{1}{\varepsilon_{0}} \boldsymbol{P}_{T}= & \sum_{\sigma=S_{j}, P_{j}}\left(1-\frac{k_{b \sigma}^{2}}{k_{0}^{2}}\right) C_{\sigma} e^{i q_{b \sigma} n} e^{i \boldsymbol{k}_{t} \cdot \boldsymbol{r}} \boldsymbol{u}_{\sigma}^{+} \\
& +C_{L} e^{i q_{b \sigma} n} e^{i \boldsymbol{k}_{t} \cdot \boldsymbol{r}} \boldsymbol{u}_{L}^{+} .
\end{aligned}
$$

The inputs of the system are the coefficients of the incident electric field $A_{S}$ and $A_{P}$, and we have to obtain the two reflected amplitudes $B_{S}$ and $B_{P}$ and the five transmitted amplitudes $C_{S 1}, C_{S 2}, C_{P 1}, C_{P 2}$, and $C_{L}$. We need, therefore, as discussed in Sec. IV A, seven equations, which correspond to the seven boundary conditions Eqs. (68a) and (68b).

It is easy to see that the $S$ polarization uncouples from the $P$ and $L$, which in turn means that the incident $A_{S}$ field excites only the $B_{S}$ reflected field and the $C_{S 1}$ and $C_{S 2}$ transmitted fields. The cancellation of the polarization vector parallel to $\boldsymbol{u}_{S}$ implies

$$
C_{S_{2}}=-\frac{k_{0}^{2}-k_{b 1}^{2}}{k_{0}^{2}-k_{b 2}^{2}} C_{S_{1}},
$$

while the continuity of the transverse components of the electric and magnetic fields gives

$$
\begin{gathered}
A_{S}+B_{S}=C_{S_{1}}+C_{S_{2}}, \\
A_{S}-B_{S}=\frac{q_{b 1}}{q_{a 0}} C_{S_{1}}+\frac{q_{b 2}}{q_{a 0}} C_{S_{2}},
\end{gathered}
$$

from which we can obtain the reflection coefficient of the $S$ mode $R_{S}=B_{S} / A_{S}$ as

$$
\frac{1-R_{S}}{1+R_{S}}=\frac{q_{b 1}}{q_{a 0}} \frac{\left(k_{b 2}^{2}-k_{0}^{2}\right)-q_{b 2} / q_{b 1}\left(k_{b 1}^{2}-k_{0}^{2}\right)}{k_{b 2}^{2}-k_{b 1}^{2}} .
$$

We can proceed similarly to obtain the reflection coefficient of the $P$ polarization $R_{P}=B_{P} / A_{P}$, using

$$
\boldsymbol{u}_{P}^{+}=-\frac{\left|\boldsymbol{k}_{t}\right|^{2}}{k_{b j}^{2}} \boldsymbol{n}+\frac{q_{b j}}{k_{b j}^{2}} \boldsymbol{k}_{t},
$$

then the continuity of the transverse electric and magnetic field is

$$
\begin{gathered}
\frac{q_{a 0}}{k_{0}^{2}}\left(A_{P}-B_{P}\right)=\frac{q_{b 1}}{k_{b 1}^{2}} C_{P_{1}}+\frac{q_{b 2}}{k_{b 2}^{2}} C_{P_{2}}+\frac{1}{k_{L}} C_{L}, \\
A_{P}+B_{P}=C_{P_{1}}+C_{P_{2}},
\end{gathered}
$$

while the cancellation of the polarization vector gives

$$
\begin{gathered}
\frac{q_{b L}}{K_{L}} C_{L}-\frac{\left|\boldsymbol{k}_{t}\right|^{2}}{k_{b 1}^{2}}\left(1-\frac{k_{b 1}^{2}}{k_{0}^{2}}\right) C_{P_{1}}-\frac{\left|\boldsymbol{k}_{t}\right|^{2}}{k_{b 2}^{2}}\left(1-\frac{k_{b 2}^{2}}{k_{0}^{2}}\right) C_{P_{2}}=0, \\
\frac{1}{K_{L}} C_{L}+\frac{q_{b 1}}{k_{b 1}^{2}}\left(1-\frac{k_{b 1}^{2}}{k_{0}^{2}}\right) C_{P_{1}}+\frac{q_{b 2}}{k_{b 2}^{2}}\left(1-\frac{k_{b 2}^{2}}{k_{0}^{2}}\right) C_{P_{2}}=0 .
\end{gathered}
$$

From the above two equations, we can obtain the relationship of the $C_{P_{1}}$ with the $C_{P_{2}}$ and $C_{L}$ coefficients, giving

$$
\begin{gathered}
C_{P_{2}}=-\frac{k_{b 2}^{2}}{k_{b 1}^{2}} \frac{q_{b L} q_{b 1}+\left|\boldsymbol{k}_{t}\right|^{2}}{q_{b L} q_{b 2}+\left|\boldsymbol{k}_{t}\right|^{2}} \frac{k_{0}^{2}-k_{b 1}^{2}}{k_{0}^{2}-k_{b 2}^{2}} C_{P_{1}} \equiv \chi_{P} C_{P_{1}}, \\
C_{L}=k_{L} \frac{q_{b 2}-q_{b 1}}{q_{b L} q_{b 2}-\left|\boldsymbol{k}_{t}\right|^{2}} \frac{\mid k^{2}\left(k_{0}^{2}-k_{b 1}^{2}\right)}{k_{0}^{2} k_{b 1}^{2}} C_{P 1} \equiv \chi_{L} C_{P_{1}},
\end{gathered}
$$

so, finally, we obtain the $R_{P}$ coefficient as

$$
\frac{1-R_{P}}{1+R_{P}}=\frac{k_{0}^{2}}{q_{a 0}} \frac{q_{b 1} / k_{b 1}^{2}+q_{b 2} / k_{b 2}^{2} \chi_{P}+1 / k_{L} \chi_{L}}{1+\chi_{P}} .
$$

At the interface between vacuum and a local dielectric material, the normal component of the electric field is not continuous and a bounded surface charge exists. This is due to the discontinuity of the polarizability of the material which induces a discontinuity in the polarization vector and, from the continuity equation,

$$
\rho=-\nabla \cdot \boldsymbol{P}=-i \boldsymbol{k} \cdot \boldsymbol{P}-\partial_{n} P_{n} \sim \delta(n),
$$

where $\delta(n)$ is the Dirac delta function. This discontinuity implies that it is actually the normal component of dielectric displacement $\boldsymbol{D}=(1+\chi) \boldsymbol{E}$ the quantity continuous through the interface. In the elastodynamic description, however, due to the continuity of the polarization vector $\boldsymbol{P}$, there are no bounded surface charges; consequently, the normal component of the electric field is also continuous at an interface.

It is interesting to remark, as was explained before, that the shear modulus in the elastodynamic description is the quantity responsible of the nonlocal response of the material at normal incidence or at oblique but with the $S$ polarization. If we ignore this term, as it is the case in the hydrodynamic model, the nonlocal response is not observed and the material is indistinguishable from a local one. This suggests that if the length scales of our system are similar to $\ell$ and we want to properly describe the spatial distribution of the fields, we should use the elastodynamic description to obtain more accurate results.

\section{SUMMARY}

In summary, a self-consistent theory for the interaction of the classical electromagnetic field and matter has been presented. The theory, developed within the framework of elastodynamics, is based on a polarization vector whose equation of motion is identical to that of the elastodynamic field, and a set of boundary conditions arise in a natural way whose number is consistent with the number of electrodynamic modes. Elementary considerations about the limiting value of the parameters of the model allow us to define vacuum, local dielectrics, real conductors, and hydrodynamic plasmas, recovering in each situation the boundary conditions employed in the literature. This description, however, includes the possibility of more advanced interfaces, composites, and symmetries, since a full anisotropic description has been considered.

This approach contains as well the basis for the consideration of more advanced phenomena, like the spill out of electrons across interfaces. We have proposed two methods to work on this issue, by considering vacuum a low-density material with some homogeneous or inhomogeneous electron density and by developing a properly defined density or current functional theories. Both approaches fits perfectly well within the present description, although further development of these is required.

Therefore, the theory presented here can be a starting point for any model of matter at the nanoscale, where the effects of spatial dispersion and continuity of the microscopic fields 
can be more relevant, and more refined models of matter are required.

This unified theory for the description of the interaction of light and matter at the nanoscale will doubtless provide a generalized description of nanophotonic structures which will be fundamental for either the characterization of nanomaterials or the accurate design of new nanophotonic devices.

\section{ACKNOWLEDGMENTS}

Daniel Torrent acknowledges financial support through the Ramón y Cajal fellowship under Grant No. RYC-2016-21188 and to the Ministry of Science, Innovation and Universities through Project No. RTI2018- 093921-A-C42. J.V.A. acknowledges financial support to the Ministry of Science, Innovation and Universities through Projects No. FIS201564886-C5-5-P and No. PGC2018-096955-B-C42.
[1] K. L. Kelly, E. Coronado, L. L. Zhao, and G. C. Schatz, J. Phys. Chem. B 107, 668 (2003).

[2] V. Amendola, R. Pilot, M. Frasconi, O. M. Marago, and M. A. Iati, J. Phys.: Condens. Matter 29, 203002 (2017).

[3] C. Burda, X. Chen, R. Narayanan, and M. A. El-Sayed, Chem. Rev. 105, 1025 (2005).

[4] C. Matricardi, C. Hanske, J. L. Garcia-Pomar, J. Langer, A. Mihi, and L. M. Liz-Marzan, ACS Nano 12, 8531 (2018).

[5] A. Espinha, C. Dore, C. Matricardi, M. I. Alonso, A. R. Goñi, and A. Mihi, Nat. Photonics 12, 343 (2018).

[6] S. Raza, S. I. Bozhevolnyi, M. Wubs, and N. A. Mortensen, J. Phys.: Condens. Matter 27, 183204 (2015).

[7] J. Aizpurua, P. Hanarp, D. S. Sutherland, M. Käll, G. W. Bryant, and F. J. Garcia de Abajo, Phys. Rev. Lett. 90, 057401 (2003).

[8] V. Myroshnychenko, J. Rodríguez-Fernández, I. PastorizaSantos, A. M. Funston, C. Novo, P. Mulvaney, L. M. Liz-Marzán, and F. J. G. de Abajo, Chem. Soc. Rev. 37, 1792 (2008).

[9] J. J. Baumberg, J. Aizpurua, M. H. Mikkelsen, and D. R. Smith, Nat. Mater. 18, 668 (2019).

[10] C. Ciraci, J. B. Pendry, and D. R. Smith, ChemPhysChem 14, 1109 (2013).

[11] J. D. Jackson, Classical Eectrodynamics (John Wiley \& sons Inc., New York, 1999).

[12] S. L. Adler, Phys. Rev. 126, 413 (1962).

[13] V. M. Agranovich and V. Ginzburg, Crystal Optics with Spatial Dispersion, and Excitons, Vol. 42 (Springer Science \& Business Media, Berlin (Germany), 2013).

[14] V. M. Agranovich and Y. N. Gartstein, Phys. Usp. 49, 1029 (2006).

[15] F. J. Garcia de Abajo, J. Phys. Chem. C 112, 17983 (2008).

[16] P. A. Belov, R. Marqués, S. I. Maslovski, I. S. Nefedov, M. Silveirinha, C. R. Simovski, and S. A. Tretyakov, Phys. Rev. B 67, 113103 (2003).

[17] C. Menzel, T. Paul, C. Rockstuhl, T. Pertsch, S. Tretyakov, and F. Lederer, Phys. Rev. B 81, 035320 (2010).

[18] A. Alù, Phys. Rev. B 83, 081102(R) (2011).

[19] A. Chipouline, C. Simovski, and S. Tretyakov, Metamaterials 6, 77 (2012).

[20] K. Mnasri, A. Khrabustovskyi, C. Stohrer, M. Plum, and C. Rockstuhl, Phys. Rev. B 97, 075439 (2018).

[21] K. Mnasri, A. Khrabustovskyi, M. Plum, and C. Rockstuhl, Phys. Rev. B 99, 035442 (2019).

[22] K. Mnasri, F. Z. Goffi, M. Plum, and C. Rockstuhl, JOSA B 36, F99 (2019).

[23] R. Rojas, F. Claro, and R. Fuchs, Phys. Rev. B 37, 6799 (1988).

[24] A. Pack, M. Hietschold, and R. Wannemacher, Opt. Commun. 194, 277 (2001).

[25] M. G. Silveirinha, IEEE Trans. Antennas Propag. 54, 1766 (2006).
[26] J. M. McMahon, S. K. Gray, and G. C. Schatz, J. Phys. Chem. C 114, 15903 (2010).

[27] S. I. Maslovski, T. A. Morgado, M. G. Silveirinha, C. S. Kaipa, and A. B. Yakovlev, New J. Phys. 12, 113047 (2010).

[28] C. David and F. J. García de Abajo, J. Phys. Chem. C 115, 19470 (2011).

[29] A. Moreau, C. Ciraci, and D. R. Smith, Phys. Rev. B 87, 045401 (2013).

[30] S. Gómez-Graña, A. Le Beulze, M. Treguer-Delapierre, S. Mornet, E. Duguet, E. Grana, E. Cloutet, G. Hadziioannou, J. Leng, J.-B. Salmon et al., Mater. Horiz. 3, 596 (2016).

[31] S. Pekar, Sov. Phys. JETP 6, 785 (1958).

[32] A. R. Melnyk and M. J. Harrison, Phys. Rev. B 2, 835 (1970).

[33] P. Halevi and R. Fuchs, J. Phys. C: Solid State Phys. 17, 3869 (1984).

[34] Y. Yang, D. Zhu, W. Yan, A. Agarwal, M. Zheng, J. D. Joannopoulos, P. Lalanne, T. Christensen, K. K. Berggren, and M. Soljačić, Nature 576, 248 (2019).

[35] K. Henneberger, Phys. Rev. Lett. 80, 2889 (1998).

[36] Y. Luo, A. I. Fernandez-Dominguez, A. Wiener, S. A. Maier, and J. B. Pendry, Phys. Rev. Lett. 111, 093901 (2013).

[37] M. Kupresak, X. Zheng, G. A. Vandenbosch, and V. V. Moshchalkov, Adv. Theor. Simul. 1, 1800076 (2018).

[38] D. Royer and E. Dieulesaint, Elastic Waves in Solids I: Free and Guided Propagation (Springer Science \& Business Media, Berlin (Germany), 1999).

[39] N. A. Mortensen, S. Raza, M. Wubs, T. Søndergaard, and S. I. Bozhevolnyi, Nat. Commun. 5, 3809 (2014).

[40] M. Fox, Optical Properties of Solids, Oxford Master Series in Physics: Condensed Matter Physics (Oxford University, 2002).

[41] I. Tokatly and O. Pankratov, Phys. Rev. B 60, 15550 (1999).

[42] S. Conti and G. Vignale, Phys. Rev. B 60, 7966 (1999).

[43] H. Goldstein, C. Poole, and J. Safko, Classical Mechanics (Classical Mechanics Addison-Wesley, Reading, MA, 1980).

[44] D. De Ceglia, M. Scalora, M. A. Vincenti, S. Campione, K. Kelley, E. L. Runnerstrom, J.-P. Maria, G. A. Keeler, and T. S. Luk, Sci. Rep. 8, 9335 (2018).

[45] M. Wubs, Opt. Express 23, 31296 (2015).

[46] I. S. Gradshteyn and I. M. Ryzhik, Table of Integrals, Series, and Products (Academic Press, Amsterdam (Netherlands), 2014).

[47] G. Toscano, J. Straubel, A. Kwiatkowski, C. Rockstuhl, F. Evers, H. Xu, N. A. Mortensen, and M. Wubs, Nat. Commun. 6, 7132 (2015).

[48] C. Ciraci and F. Della Sala, Phys. Rev. B 93, 205405 (2016).

[49] K. Ding and C. Chan, J. Phys.: Condens. Matter 30, 084007 (2018).

[50] C. Ciracì, Phys. Rev. B 95, 245434 (2017).

[51] G. Grosso, Solid State Physics, 2nd ed. (British Library Cataloguing, UK, 2003). 